\title{
Towards Euclidean theory of infrared singular quantum fields
}

\author{
A. G. Smirnov* \\ I. E. Tamm Theory Department, P. N. Lebedev Physical Institute, \\ Leninsky prospect 53, Moscow 119991, Russia
}

\begin{abstract}
A new generalized formulation of the spectral condition is proposed for quantum fields with highly singular infrared behavior whose vacuum correlation functions are well defined only under smearing with analytic test functions in momentum space. The Euclidean formulation of QFT developed by Osterwalder and Schrader is extended to theories with infrared singular indefinite metric. The corresponding generalization of the reconstruction theorem is obtained. The fulfilment of the generalized spectral condition is verified for quantum fields representable by infinite series in the Wick powers of indefinite metric free fields.
\end{abstract}

*E-mail: smirnov@lpi.ru 


\section{Introduction}

The Euclidean methods are central to the rigorous construction of quantum field models with polynomial interaction in lower dimensions, see [1]. This construction heavily relies on the use of Osterwalder-Schrader reconstruction theorem [2, 3] which allows to pass from Euclidean Green's functions to quantum field theory in the Minkowski spacetime. However, the results of [2, 3] in their initial form are inapplicable to models with a singular infrared behavior violating the positivity condition and, in particular, to gauge theories. The problem of the Euclidean formulation of QFT in the case of pseudo-Wightman axioms with an indefinite metric [4, 5] was considered in [6] within the traditional framework of tempered Schwartz distributions. However, as shown, in particular, by the example of the Schwinger model in an arbitrary $\alpha$-gauge [7], the exact operator solutions of gauge models can be much more singular and, in general, are well defined only under smearing with analytic test functions in momentum space. In this work, we study the possibility of extending the Euclidean theory to the fields whose vacuum expectation values are analytic functionals in momentum representation.

One of the main difficulties is connected with the adequate generalization of the spectral condition, which determines the analyticity properties of the Wightman functions. In indefinite metric field theories, the space-time translations are implemented by pseudo-unitary (in general, unbounded) operators in the state space and, therefore, the spectral condition can be formulated only in the weak form, i.e., as a restriction on the $n$-point Wightman functions $w_{n}$. When $w_{n}$ are tempered distributions, it is of the same form as in the ordinary Wightman theory: ${ }^{1}$

$$
\operatorname{supp} \hat{W}_{n} \subset \overline{\mathbb{V}}_{+}^{n}, \quad \hat{W}_{n}\left(p_{1}, \ldots, p_{n}\right)=\int W_{n}\left(\xi_{1}, \ldots, \xi_{n}\right) e^{i p_{1} \xi_{1}+\ldots+i p_{n} \xi_{n}} \mathrm{~d} p_{1} \ldots \mathrm{d} p_{n},
$$

where $\overline{\mathbb{V}}_{+}$is the closed upper light cone and $W_{n}(\xi)$ is the Wightman function $w_{n+1}$ expressed in terms of the difference variables $\xi_{j}=x_{j}-x_{j+1}$ :

$$
w_{n+1}\left(x_{1}, \ldots, x_{n+1}\right)=W_{n}\left(x_{1}-x_{2}, \ldots, x_{n}-x_{n+1}\right) .
$$

If $\hat{W}_{n}$ are analytic functionals, then the condition (1D) becomes inapplicable because of the lack of test functions with compact support. The problem of the appropriate generalization of the spectral condition was raised by Moschella and Strocchi [8]. In [9], it was suggested to overcome this difficulty using the notion of carrier cone which replaces the notion of support for analytic functionals and whose existence for a wide class of functionals was proved in [10, 11. The generalized spectral condition which is obtained from (11) by replacing the support with a carrier cone is sufficient for deriving the usual analyticity properties of the Wightman functions [9] and is fulfilled for the sums of infinite series in the Wick powers of indefinite metric free fields [12]. Moreover, fields representable by such series satisfy even stronger condition stated in terms of the

\footnotetext{
${ }^{1}$ To avoid inessential technical complications, we consider the case of a single scalar field in $d$ dimensional space-time $(d \geq 2)$.
} 
notion of strong carrier cone which is introduced by Definition 2 below. The latter arises naturally when one tries to bring the definition of carrier cone into line with with the operation of tensor product of functionals, which plays an important role in the problem under consideration. The use of the generalized spectral condition in such a stronger form yields simple and effective estimates for the Schwinger functions which allow developing the Euclidean formulation in complete analogy with the case of tempered fields [3]. In this paper, the analysis of the Euclidean formulation of QFT is performed at the level of the Wightman functions of the theory. At the same time, we believe that the developed approach provides a basis for considering more subtle questions connected with finding the Hilbert majorant of an indefinite metric [4].

As in [8]-[11, we use the Gelfand-Shilov spaces $S_{\beta}^{\alpha}$ as the functional domain of definition of fields in momentum space. The generalized functions belonging to $S_{\beta}^{\prime \alpha} 2$ grow at infinity like $\exp \left(|p|^{1 / \beta}\right)$ and their Fourier-transforms like $\exp \left(|x|^{1 / \alpha}\right)$. Thus, the indices $\alpha$ and $\beta$ control, respectively, the possible infrared and ultraviolet singularities. If $0 \leq \alpha<1$, then the elements of $S_{\beta}^{\alpha}$ are entire analytic functions. It is important that our treatment covers the case $\alpha=0$ which corresponds to an arbitrary singular infrared behavior.

In Sec. 2. we introduce the definition of strong carrier cone and prove that the intersection of strong carrier cones of a functional is again its strong carrier cone. Analogous result for carrier cones ensuring, in particular, the existence of the smallest carrier cone was established in [10. In Sec. 3. we prove that the definition of strong carrier cone is compatible with the operation of tensor product of functionals. In Sec. 固 the theory of Laplace transformation is extended to functionals with convex strong carrier cones. In particular, we prove a Paley-Wiener-Schwartz type theorem characterizing those analytic functions that are Laplace transforms of such functionals. In Sec. 5 , this theorem is applied to derive estimates for the Schwinger functions. In the same section the main result of the paper is presented, namely, the generalized Euclidean reconstruction theorem which covers field theories with arbitrarily singular infrared behavior. In Sec. 6 we check that the generalized spectral condition is satisfied for normally ordered entire functions of indefinite metric free fields. Sec. 7 is devoted to concluding remarks. Some details of proofs are given in Appendices A and B.

\section{Strong carrier cones}

The space $S_{\beta}^{\alpha}\left(\mathbb{R}^{k}\right)$ is by definition [13 the union (inductive limit) with respect to $A, B>$ 0 of the Banach spaces composed of smooth functions on $\mathbb{R}^{k}$ with the finite norm

$$
\left.|||f|\right|_{A, B}=\sup _{p \in \mathbb{R}^{k}, \lambda, \mu} \frac{\left|p^{\mu} \partial^{\lambda} f(p)\right|}{A^{|\lambda|} B^{|\mu|}|\lambda|^{\alpha|\lambda|}|\mu|^{|\beta| \mu \mid}},
$$

\footnotetext{
${ }^{2}$ Here and subsequently, we denote the continuous dual of a topological vector space by the same symbol with a prime.
} 
where $\lambda$ and $\mu$ run over all multi-indices and the standard multi-index notation is used. The spaces $S_{\beta}^{\alpha}$ are nontrivial if $\alpha+\beta>1$ or if $\alpha>0$ and $\alpha+\beta=1$. From now on, we assume that one of these conditions is satisfied. If $0 \leq \alpha<1$, then $S_{\beta}^{\alpha}$ consists of (the restrictions to $\mathbb{R}^{k}$ of) entire analytic functions and an alternative description of these spaces in terms of complex variables is possible [13]. Namely, an analytic function $f$ on $\mathbb{C}^{k}$ belongs to the class $S_{\beta}^{\alpha}$ if and only if

$$
|f(w)| \leq C \exp \left(-|p / B|^{1 / \beta}+|A q|^{1 /(1-\alpha)}\right), \quad w=p+i q \in \mathbb{C}^{k},
$$

for some $A, B>0$ depending on $f$. For definiteness, we assume the norm $|\cdot|$ on $\mathbb{R}^{k}$ to be uniform, i.e., $|p|=\sup _{1 \leq j \leq k}\left|p_{j}\right|$. The main element of the approach developed in [10, 11] is the employment, in addition to the spaces $S_{\beta}^{\alpha}$, of analogous spaces associated with cones.

Definition 1. Let $U$ be a nonempty cone in $\mathbb{R}^{k}$. The Banach space $S_{\beta, B}^{\alpha, A}(U)$, $0 \leq \alpha<1$, consists of entire analytic functions on $\mathbb{C}^{k}$ with the finite norm

$$
\|f\|_{U, A, B}=\sup _{w \in \mathbb{C}^{k}}|f(w)| \exp \left(|p / B|^{1 / \beta}-|A q|^{1 /(1-\alpha)}-\delta_{U}(A p)^{1 /(1-\alpha)}\right),
$$

where $\delta_{U}(p)=\inf _{p^{\prime} \in U}\left|p-p^{\prime}\right|$. The space $S_{\beta}^{\alpha}(U)$ is defined to be the inductive limit $\lim _{A, B>0} S_{\beta, B}^{\alpha, A}(U)$.

A nonempty closed cone $K$ is called a carrier cone of the functional $u \in S_{\beta}^{\prime \alpha}\left(\mathbb{R}^{k}\right)$ if $u$ extends continuously to every space $S_{\beta}^{\alpha}(U)$, where $U$ is a cone with an open projection ${ }^{3}$ such that $K \subset U$. As shown in [10, 11, the space $S_{\beta}^{\alpha}\left(\mathbb{R}^{k}\right)$ is dense in each space $S_{\beta}^{\alpha}(U)$. The space of the functionals carried by the cone $K$ is therefore identified with $s_{\beta}^{\prime \alpha}(K)$, where $s_{\beta}^{\alpha}(K)=\lim _{U} S_{\beta}^{\alpha}(U)$.

It should be mentioned that in 10, 11, the spaces $S_{\beta}^{\alpha}(U)$ are defined for open cones $U$ and a closed cone $K$ is said to be a carrier cone of $u$ if this functional has a continuous extension to every $S_{\beta}^{\alpha}(U)$, where $K \backslash\{0\} \subset U$. This definition is equivalent to the one given here. It is easy to see that all results of [10, 11] concerning the spaces $S_{\beta}^{\alpha}(U)$ remain true for any nonempty cone $U$. In what follows, we find it convenient to use the spaces $S_{\beta}^{\alpha}(U)$ associated with arbitrary nonempty cones because this allows handling the degenerate cone $\{0\}$ on the same footing as nondegenerate closed carrier cones. We also note that in [10, 11], the space $s_{\beta}^{\alpha}(K)$ was denoted by $S_{\beta}^{\alpha}(K)$. Here, such notation might lead to confusion because the spaces $S_{\beta}^{\alpha}(K)$ and $S_{\beta}^{\alpha}(U)$ are no longer distinguished by the type of the cone.

The following result established in [10] shows that every functional of the class $S_{\beta}^{\prime \alpha}\left(\mathbb{R}^{k}\right)$ has a uniquely defined minimal carrier cone.

Theorem 1. If both $K_{1}$ and $K_{2}$ are carrier cones of $u \in S_{\beta}^{\prime \alpha}\left(\mathbb{R}^{n}\right)$, then so is $K_{1} \cap K_{2}$.

The cone $\overline{\mathbb{V}}_{+}^{n}$, which enters into the formulation (II) of the spectral condition, has a natural direct product structure and the following definition turns out to be useful for generalizing the spectral condition.

\footnotetext{
${ }^{3}$ The projection of the cone $U$ is by definition the intersection of $U$ with the unit sphere in $\mathbb{R}^{k}$; the projection of $U$ is meant to be open in the topology of the sphere.
} 
Definition 2. Let $K_{1}, \ldots, K_{n}$ be nonempty closed cones in $\mathbb{R}^{k_{1}}, \ldots, \mathbb{R}^{k_{n}}$ respectively. The cone $K_{1} \times \ldots \times K_{n}$ is called a strong carrier cone of the functional $u \in S_{\beta}^{\prime \alpha}\left(\mathbb{R}^{k_{1}+\ldots+k_{n}}\right)$ if $u$ allows a continuous extension to the space $s_{\beta}^{\alpha}\left(K_{1}, \ldots, K_{n}\right)=$ $\lim _{U_{1}, \ldots, U_{n}} S_{\beta}^{\alpha}\left(U_{1} \times \ldots \times U_{n}\right)$, where the inductive limit is taken over all cones $U_{1}, \ldots, U_{n}$ with open projections such that $K_{j} \subset U_{j}$ for all $j=1, \ldots, n$.

The meaning of the spaces $s_{\beta}^{\prime \alpha}\left(K_{1}, \ldots, K_{n}\right)$ is clarified by Lemma 5 below. If $n=1$, then we recover the definition of carrier cone. As shown in [11, the natural embeddings $S_{\beta, B}^{\alpha, A}(U) \rightarrow S_{\beta, B^{\prime}}^{\alpha, A^{\prime}}(U)$ are compact for $A^{\prime}>A, B^{\prime}>B$ sufficiently large. Therefore, $S_{\beta}^{\alpha}(U)$ and $s_{\beta}^{\alpha}\left(K_{1}, \ldots, K_{n}\right)$ are DFS-spaces (we recall that DFS-spaces are, by definition, the inductive limits of injective compact sequences of locally convex spaces). In particular, they (and their duals) are reflexive, complete, and Montel spaces [14.

Clearly, $s_{\beta}^{\prime \alpha}\left(K_{1}, \ldots, K_{n}\right) \subset s_{\beta}^{\prime \alpha}\left(K_{1} \times \ldots \times K_{n}\right)$, but the following example shows that the condition $u \in s_{\beta}^{\alpha}\left(K_{1}, \ldots, K_{n}\right)$ is, in general, stronger than the condition $u \in$ $s_{\beta}^{\prime \alpha}\left(K_{1} \times \ldots \times K_{n}\right)$.

Example 1. Let $u(p)$ be the function equal to unity on the set $\left\{p \in \mathbb{R}^{2} \mid p_{2} \geq\right.$ $\left.-\left|p_{1}\right|^{2 / 3}\right\}$ and zero outside this set. As a generalized function, $u$ obviously belongs to $s_{\beta}^{\prime \alpha}\left(\mathbb{R} \times \overline{\mathbb{R}}_{+}\right)$for all $0 \leq \alpha<1$ and $\beta>1-\alpha$. Let us show that $u \notin s_{1 / 2}^{\prime 2 / 3}\left(\mathbb{R}, \overline{\mathbb{R}}_{+}\right)=$ $S_{1 / 2}^{\prime 2 / 3}\left(\mathbb{R} \times \mathbb{R}_{+}\right)$. Use the test function $f(w)=\exp \left(-w_{1}^{2}-w_{2}^{3}\right)$ belonging to $S_{1 / 2}^{2 / 3}\left(\mathbb{R} \times \mathbb{R}_{+}\right)$ and define $g(w)$ by the same formula as $f$ but with the twice less exponent. By the above-mentioned density property, there exists a sequence $g_{\nu} \in S_{1 / 2}^{2 / 3}\left(\mathbb{R}^{2}\right)$ converging to $g$ in $S_{1 / 2}^{2 / 3}\left(\mathbb{R} \times \mathbb{R}_{+}\right)$. Set $f_{\nu}(w)=g_{\nu}(w) \overline{g_{\nu}(\bar{w})}$ (bar means complex conjugation). Obviously, $f_{\nu}(p) \geq 0$ and $f_{\nu} \rightarrow f$ in $S_{1 / 2}^{2 / 3}\left(\mathbb{R} \times \mathbb{R}_{+}\right)$. Since the integral $\int u(p) f(p) \mathrm{d} p$ is divergent, we conclude by the monotonic convergence theorem that $\int u(p) f_{\nu}(p) \mathrm{d} p \rightarrow \infty$ as $\nu \rightarrow \infty$.

The following analogue of Theorem 1 is valid.

Theorem 2. Let $K_{1}^{(1,2)}, \ldots, K_{n}^{(1,2)}$ be nonempty closed cones in $\mathbb{R}^{k_{1}}, \ldots, \mathbb{R}^{k_{n}}$ respectively. If both $K_{1}^{(1)} \times \ldots \times K_{n}^{(1)}$ and $K_{1}^{(2)} \times \ldots \times K_{n}^{(2)}$ are strong carrier cones of $u \in S_{\beta}^{\prime \alpha}\left(\mathbb{R}^{k_{1}+\ldots+k_{n}}\right)$, then so is $\left(K_{1}^{(1)} \cap K_{1}^{(2)}\right) \times \ldots \times\left(K_{n}^{(1)} \cap K_{n}^{(2)}\right)$.

Before we pass to the proof, let us set up the notation and recall some facts concerning cones in $\mathbb{R}^{k}$. Let $\mathcal{C}\left(\mathbb{R}^{k}\right)$ denote the set of all cones in $\mathbb{R}^{k}$ containing the origin and let $\mathcal{O}\left(\mathbb{R}^{k}\right)$ be the subset of $\mathcal{C}\left(\mathbb{R}^{k}\right)$ consisting of cones with open projections (or, which is the same, of those cones whose intersection with $\mathbb{R}^{k} \backslash\{0\}$ is open). We note that the cones $U_{j}$ in Definition 2 belong to $\mathcal{O}\left(\mathbb{R}^{k_{j}}\right)$. Obviously, for any (open) subset $O$ of the unit sphere there is a unique cone $U \in \mathcal{C}\left(\mathbb{R}^{k}\right)$ (resp., $U \in \mathcal{O}\left(\mathbb{R}^{k}\right)$ ) such that $O$ is the projection of $U$. Using this one-to-one correspondence, one can apply standard compactness arguments to cones in $\mathcal{C}\left(\mathbb{R}^{k}\right)$ to obtain:

(I) if $U \in \mathcal{C}\left(\mathbb{R}^{k}\right), V \in \mathcal{O}\left(\mathbb{R}^{k}\right)$, and $U \Subset V,{ }^{4}$ then there exists $W \in \mathcal{O}\left(\mathbb{R}^{k}\right)$ such that $U \Subset W \Subset V ;$

(II) if $U_{1}, U_{2} \in \mathcal{C}\left(\mathbb{R}^{k}\right)$ and $\bar{U}_{1} \cap \bar{U}_{2}=\{0\}$, then there exist $V_{1}, V_{2} \in \mathcal{O}\left(\mathbb{R}^{k}\right)$ such that $U_{1,2} \Subset V_{1,2}$ and $\bar{V}_{1} \cap \bar{V}_{2}=\{0\} ;$

\footnotetext{
${ }^{4} \mathrm{~A}$ cone $U$ is said to be compact in a cone $V$ (notation $\left.U \Subset V\right)$ if $\bar{U} \backslash\{0\} \subset V$.
} 
(III) if $V \in \mathcal{C}\left(\mathbb{R}^{k}\right), U \in \mathcal{O}\left(\mathbb{R}^{k}\right)$, and $V \Subset U$, then $\bar{V} \cap \Delta U=\{0\}$, where $\Delta U=$ $\left(\mathbb{R}^{k} \backslash U\right) \cup\{0\}$ corresponds to the complement of the projection of $U$ in the unit sphere.

Proof of Theorem 圆 Let $u_{1,2}$ be the extensions of $u$ to the spaces $s_{\beta}^{\alpha}\left(K_{1}^{(1,2)}, \ldots, K_{n}^{(1,2)}\right)$ which exist by the hypothesis and let $f \in s_{\beta}^{\alpha}\left(K_{1}^{(1)}, \ldots, K_{n}^{(1)}\right) \cap s_{\beta}^{\alpha}\left(K_{1}^{(2)}, \ldots, K_{n}^{(2)}\right)$. By Definition 2, there are cones $U_{1}^{(1,2)} \in \mathcal{O}\left(\mathbb{R}^{k_{1}}\right), \ldots, U_{n}^{(1,2)} \in \mathcal{O}\left(\mathbb{R}^{k_{n}}\right)$ such that $K_{1}^{(1,2)} \times \ldots \times$ $K_{n}^{(1,2)} \subset V^{(1,2)}=U_{1}^{(1,2)} \times \ldots \times U_{n}^{(1,2)}$ and $f \in S_{\beta}^{\alpha}\left(V^{(1)}\right) \cap S_{\beta}^{\alpha}\left(V^{(2)}\right)=S_{\beta}^{\alpha}\left(V^{(1)} \cup V^{(2)}\right)$. The existence of continuous dense embeddings $S_{\beta}^{\alpha}\left(\mathbb{R}^{k_{1}+\ldots+k_{n}}\right) \rightarrow S_{\beta}^{\alpha}\left(V^{(1)} \cup V^{(2)}\right) \rightarrow$ $s_{\beta}^{\alpha}\left(K_{1}^{(1,2)}, \ldots, K_{n}^{(1,2)}\right)$ implies that $u_{1}$ and $u_{2}$ coincide on $S_{\beta}^{\alpha}\left(V^{(1)} \cup V^{(2)}\right)$ and, consequently,

$$
u_{1}(f)=u_{2}(f) \text { for every } f \in s_{\beta}^{\alpha}\left(K_{1}^{(1)}, \ldots, K_{n}^{(1)}\right) \cap s_{\beta}^{\alpha}\left(K_{1}^{(2)}, \ldots, K_{n}^{(2)}\right) .
$$

Let us consider the mapping

$$
j: s_{\beta}^{\alpha}\left(K_{1}^{(1)}, \ldots, K_{n}^{(1)}\right) \times s_{\beta}^{\alpha}\left(K_{1}^{(2)}, \ldots, K_{n}^{(2)}\right) \rightarrow s_{\beta}^{\alpha}\left(K_{1}^{(1)} \cap K_{1}^{(2)}, \ldots, K_{n}^{(1)} \cap K_{n}^{(2)}\right)
$$

taking $\left(f_{1}, f_{2}\right)$ to $f_{1}-f_{2}$. If $j$ is surjective, then $s_{\beta}^{\alpha}\left(K_{1}^{(1)} \cap K_{1}^{(2)}, \ldots, K_{n}^{(1)} \cap K_{n}^{(2)}\right)$ is topologically isomorphic to the quotient space $\left[s_{\beta}^{\alpha}\left(K_{1}^{(1)}, \ldots, K_{n}^{(1)}\right) \times s_{\beta}^{\alpha}\left(K_{1}^{(2)}, \ldots, K_{n}^{(2)}\right)\right] / \operatorname{ker} j$ by the open mapping theorem (see [15], Theorem IV.8.3.), which is applicable because all spaces under consideration are DFS. From (5), it follows that ker $j$ is contained in the kernel of the functional $\left(f_{1}, f_{2}\right) \rightarrow u_{1}\left(f_{1}\right)+u_{2}\left(f_{2}\right)$. As a consequence, the latter allows a canonical decomposition of the form $\tilde{u} \circ j$, where $\tilde{u}$ belongs to $s_{\beta}^{\prime \alpha}\left(K_{1}^{(1)} \cap K_{1}^{(2)}, \ldots, K_{n}^{(1)} \cap K_{n}^{(2)}\right)$ and, as one can easily see, is the extension of $u$. Thus, it remains to prove the surjectivity of $j$. It is ensured by the following decomposition theorem for test functions.

Theorem 3. If $f \in s_{\beta}^{\alpha}\left(K_{1}^{(1)} \cap K_{1}^{(2)}, \ldots, K_{n}^{(1)} \cap K_{n}^{(2)}\right)$ then $f=f^{(1)}+f^{(2)}$ with $f^{(1,2)} \in s_{\beta}^{\alpha}\left(K_{1}^{(1,2)}, \ldots, K_{n}^{(1,2)}\right)$.

To prove Theorem 3, we need three lemmas.

Lemma 1. Let $U \in \mathcal{C}\left(\mathbb{R}^{k_{1}}\right), V \in \mathcal{C}\left(\mathbb{R}^{k_{2}}\right)$, and let $U_{1}, U_{2} \in \mathcal{C}\left(\mathbb{R}^{k_{1}}\right)$ be such that $\bar{U}_{1} \cap \bar{U}_{2}=\{0\}$. Then for every $f \in S_{\beta}^{\alpha}(U \times V)$ one can find $f_{1,2} \in S_{\beta}^{\alpha}\left(\left(U \cup U_{1,2}\right) \times V\right)$ such that $f=f_{1}+f_{2}$. If $U \in \mathcal{O}\left(\mathbb{R}^{k_{1}}\right)$, then the condition $\bar{U}_{1} \cap \bar{U}_{2}=\{0\}$ can be replaced by $\bar{U}_{1} \cap \bar{U}_{2} \subset U$.

Proof will be given for $0<\alpha<1$, when the space $S_{1-\alpha}^{\alpha}$ is nontrivial. The more difficult case $\alpha=0$ is considered in Appendix A. By (II), there exist cones $Q_{1}, Q_{2} \in$ $\mathcal{O}\left(\mathbb{R}^{k_{1}}\right)$ such that $U_{1,2} \Subset Q_{1,2}$ and $\bar{Q}_{1} \cap \bar{Q}_{2}=\{0\}$, and in view of (I) one can find cones $V_{1}, V_{2} \in \mathcal{O}\left(\mathbb{R}^{k_{1}}\right)$ such that $U_{1,2} \Subset V_{1,2} \Subset Q_{1,2}$. Set $W_{1}=Q_{1}$ and $W_{2}=\Delta Q_{1}$. By (III) we have $\bar{W}_{1} \cap \bar{V}_{2}=\bar{V}_{1} \cap \bar{W}_{2}=\{0\}$. Let $g_{0} \in S_{1-\alpha, B_{0}}^{\alpha, A_{0}}\left(\mathbb{R}^{k_{1}}\right)$ and $\int_{\mathbb{R}^{k_{1}}} g_{0}\left(p^{\prime}\right) \mathrm{d} p^{\prime}=1$. We set

$$
g_{1,2}(w)=\int_{W_{2,1}} g_{0}\left(w^{\prime}-\eta\right) \mathrm{d} \eta, \quad w=\left(w^{\prime}, w^{\prime \prime}\right) \in \mathbb{C}^{k_{1}} \times \mathbb{C}^{k_{2}} .
$$


Obviously, $g_{1}, g_{2}$ are entire analytic functions on $\mathbb{C}^{k_{1}} \times \mathbb{C}^{k_{2}}$ and $g_{1}+g_{2}=1$. If $\eta \in W_{1,2}$, then $\left|p^{\prime}-\eta\right| \geq \delta_{W_{1,2}}\left(p^{\prime}\right)$ and in view of (4) we have

$$
\left|g_{1,2}(w)\right| \leq C \exp \left[\left(A_{0}\left|q^{\prime}\right|\right)^{1 /(1-\alpha)}-\delta_{W_{2,1}}\left(\frac{p^{\prime}}{2 B_{0}}\right)^{1 /(1-\alpha)}\right]
$$

Set $f_{1,2}=f g_{1,2}$ and fix $A, B>0$ such that $f \in S_{\beta, B}^{\alpha, A}(U \times V)$. If $p^{\prime} \notin V_{1}$, then $\delta_{U_{1}}\left(p^{\prime}\right) \geq$ $\theta\left|p^{\prime}\right|$ for some $0<\theta<1$ and in view of the inequality $\left|p^{\prime}\right| \geq \delta_{U}\left(p^{\prime}\right)$ we have $\delta_{U}\left(p^{\prime}\right) \leq$ $\delta_{U_{1} \cup U}\left(p^{\prime} / \theta\right)$. Hence, taking (44), (17) and the relation $\delta_{U \times V}(p)=\max \left[\delta_{U}\left(p^{\prime}\right), \delta_{V}\left(p^{\prime \prime}\right)\right]$ into account, we find that

$$
\left|f_{1}(w)\right| \leq C\|f\|_{A, B} \exp \left[\left(2\left(A_{0}+A\right)|q|\right)^{\frac{1}{1-\alpha}}+\delta_{\left(U_{1} \cup U\right) \times V}(A p / \theta)^{\frac{1}{1-\alpha}}-(|p| / B)^{1 / \beta}\right]
$$

for $p^{\prime} \notin V_{1}$. Further, there is $\theta_{1}>0$ such that $\delta_{W_{2}}\left(p^{\prime}\right) \geq \theta_{1}\left|p^{\prime}\right|$ for $p^{\prime} \in V_{1}$. Therefore, for $A \leq \theta_{1} / 2 B_{0}$, using (4) and (17), we obtain

$$
\left|f_{1}(w)\right| \leq C\|f\|_{A, B} \exp \left[\left(2\left(A_{0}+A\right)|q|\right)^{\frac{1}{1-\alpha}}+\delta_{V}\left(A p^{\prime \prime}\right)^{\frac{1}{1-\alpha}}-(|p| / B)^{1 / \beta}\right]
$$

for $p^{\prime} \in V_{1}$. Comparing (8) and (9)), we conclude that $f_{1} \in S_{\beta, B}^{\alpha, A^{\prime}}\left(\left(U \cup U_{1}\right) \times V\right)$ for $A^{\prime} \geq 2\left(A_{0}+A\right)+A / \theta$. Analogous arguments show that $f_{2} \in S_{\beta}^{\alpha}\left(\left(U \cup U_{2}\right) \times V\right)$ for $B_{0}$ sufficiently large.

If $U \in \mathcal{O}\left(\mathbb{R}^{k_{1}}\right)$, then $K_{1} \cap K_{2}=\{0\}$ for the nonempty closed cones $K_{1,2}=\bar{U}_{1,2} \cap \Delta U$, and according to the above we have a decomposition $f=f_{1}+f_{2}$, where $f_{1,2} \in S_{\beta}^{\alpha}((U \cup$ $\left.\left.K_{1,2}\right) \times V\right)$. To complete the proof, it remains to note that $K_{1,2} \cup U \supset U_{1,2} \cup U$.

Lemma 2. Let $U_{1} \in \mathcal{O}\left(\mathbb{R}^{k_{1}}\right), U_{2} \in \mathcal{O}\left(\mathbb{R}^{k_{2}}\right), U \in \mathcal{C}\left(\mathbb{R}^{k}\right)$, and $V_{1,2} \in \mathcal{C}\left(\mathbb{R}^{k_{1,2}}\right)$ be such that $V_{1,2} \Subset U_{1,2}$. Then for every $f \in S_{\beta}^{\alpha}\left(U_{1} \times U_{2} \times U\right)$, there is a decomposition $f=f_{1}+f_{2}$, where $f_{1} \in S_{\beta}^{\alpha}\left(V_{1} \times \mathbb{R}^{k_{2}} \times U\right)$ and $f_{2} \in S_{\beta}^{\alpha}\left(\mathbb{R}^{k_{1}} \times V_{2} \times U\right)$.

Proof. By (I), one can find $W_{1,2} \in \mathcal{O}\left(\mathbb{R}^{k_{1,2}}\right)$ such that $V_{1,2} \Subset W_{1,2} \Subset U_{1,2}$. Set $Q_{1}=\bar{V}_{1} \times \Delta W_{2}$ and $Q_{2}=\Delta W_{1} \times \bar{V}_{2}$. According to (III) we have $\bar{Q}_{1} \cap \bar{Q}_{2}=\{0\}$ and by Lemma 1, $f=f_{1}+f_{2}$, where $f_{1,2} \in S_{\beta}^{\alpha}\left(\left[\left(U_{1} \times U_{2}\right) \cup Q_{1,2}\right] \times U\right)$. It remains to note that $\left(U_{1} \times U_{2}\right) \cup Q_{1} \supset V_{1} \times \mathbb{R}^{k_{2}}$ and $\left(U_{1} \times U_{2}\right) \cup Q_{2} \supset \mathbb{R}^{k_{1}} \times V_{2}$.

Lemma 3. Let $V_{1}, U_{1}, \ldots, V_{n}, U_{n}$ be cones in $\mathbb{R}^{k_{1}}, \ldots, \mathbb{R}^{k_{n}}$ such that $V_{j} \in \mathcal{C}\left(\mathbb{R}^{k_{j}}\right)$, $U_{j} \in \mathcal{O}\left(\mathbb{R}^{k_{j}}\right)$, and $V_{j} \Subset U_{j}$ for all $j=1, \ldots, n$. If $f \in S_{\beta}^{\alpha}\left(U_{1} \times \ldots \times U_{n}\right)$, then $f=f_{1}+\ldots+f_{n}$ with $f_{j} \in S_{\beta}^{\alpha}\left(\mathbb{R}^{k_{1}} \times \ldots \times \mathbb{R}^{k_{j-1}} \times V_{j} \times \mathbb{R}^{k_{j+1}} \times \ldots \times \mathbb{R}^{k_{n}}\right)$.

Proof. We shall prove the following stronger statement. Let the cones $V_{1}, U_{1}, \ldots, V_{n}, U_{n}$ satisfy the conditions of the lemma and let $U \in \mathcal{C}\left(\mathbb{R}^{k}\right)$. Then for every $f \in S_{\beta}^{\alpha}\left(U_{1} \times \ldots \times\right.$ $\left.U_{n} \times U\right)$ there exists a decomposition $f=f_{1}+\ldots+f_{n}$, where $f_{j} \in S_{\beta}^{\alpha}\left(\mathbb{R}^{k_{1}} \times \ldots \times \mathbb{R}^{k_{j-1}} \times\right.$ $\left.V_{j} \times \mathbb{R}^{k_{j+1}} \times \ldots \times \mathbb{R}^{k_{n}} \times U\right)$. The statement of the lemma corresponds to the particular case $\mathbb{R}^{k}=U=\{0\}$. For $n=2$, the proof is reduced to applying Lemma 2. For $n>2$, we proceed by induction on $n$. Supposing the statement to hold up to $n-1$, we choose the cones $W_{1,2} \in \mathcal{O}\left(\mathbb{R}^{k_{1,2}}\right)$ such that $V_{1,2} \Subset W_{1,2} \Subset U_{1,2}$. By Lemma 2, $f=\tilde{f}_{1}+\tilde{f}_{2}$, where $\tilde{f}_{1} \in S_{\beta}^{\alpha}\left(W_{1} \times \mathbb{R}^{k_{2}} \times U_{3} \times \ldots \times U_{n} \times U\right)$ and $\tilde{f}_{2} \in S_{\beta}^{\alpha}\left(\mathbb{R}^{k_{1}} \times W_{2} \times U_{3} \times \ldots \times U_{n} \times U\right)$, and in view 
of the natural isomorphisms $W_{1} \times \mathbb{R}^{k_{2}} \times U_{3} \times \ldots \times U_{n} \times U \cong W_{1} \times U_{3} \times \ldots \times U_{n} \times\left(\mathbb{R}^{k_{2}} \times U\right)$ and $\mathbb{R}^{k_{1}} \times W_{2} \times U_{3} \times \ldots \times U_{n} \times U \cong W_{2} \times U_{3} \times \ldots \times U_{n} \times\left(\mathbb{R}^{k_{1}} \times U\right)$ we obtain the desired decompositions of $\tilde{f}_{1}$ and $\tilde{f}_{2}$. The lemma is proved.

We now prove Theorem 3, By Lemma 3, we have a decomposition $f=f_{1}+\ldots+f_{n}$, where $f_{j} \in s_{\beta}^{\alpha}\left(\mathbb{R}^{k_{1}}, \ldots, \mathbb{R}^{k_{j-1}}, K_{j}^{(1)} \cap K_{j}^{(2)}, \mathbb{R}^{k_{j+1}}, \ldots, \mathbb{R}^{k_{n}}\right), j=1, \ldots, n$. Let the cones $U_{j}^{(1)}, U_{j}^{(2)}, U_{j} \in \mathcal{O}\left(\mathbb{R}^{k_{j}}\right)$ be such that $f_{j} \in S_{\beta}^{\alpha}\left(\mathbb{R}^{k_{1}}, \ldots, \mathbb{R}^{k_{j-1}}, U_{j}, \mathbb{R}^{k_{j+1}}, \ldots, \mathbb{R}^{k_{n}}\right), K_{j}^{(1,2)} \Subset$ $U_{j}^{(1,2)}$ and $\bar{U}_{j}^{(1)} \cap \bar{U}_{j}^{(2)} \subset U_{j}$. By Lemma 1 there is a decomposition $f_{j}=f_{j}^{(1)}+f_{j}^{(2)}$, where $f_{j}^{(1,2)} \in S_{\beta}^{\alpha}\left(\mathbb{R}^{k_{1}} \times \ldots \times \mathbb{R}^{k_{j-1}} \times U_{j}^{(1,2)} \times \mathbb{R}^{k_{j+1}} \times \ldots \times \mathbb{R}^{k_{n}}\right)$. Setting $f^{(1,2)}=f_{1}^{(1,2)}+\ldots+f_{n}^{(1,2)}$, we arrive at the desired result.

\section{$3 \quad$ Tensor products}

We refer the reader to [15] for the definition and properties of the inductive topology ( $i$-topology), projective topology ( $\pi$-topology), and the topology of equicontinuous convergence (e-topology) on tensor products of locally convex spaces. Recall that $\pi$ - and $e$-topologies coincide on the tensor products of nuclear spaces, while $i$ - and $\pi$-topologies coincide on the tensor products of Fréchet spaces.

Lemma 4. Let $L^{(1)}$ and $L^{(2)}$ be DFS-spaces. Then $L^{(1)} \otimes_{i} L^{(2)}=L^{(1)} \otimes_{\pi} L^{(2)}$. If $L^{(1)}$ is nuclear, then $\left(L^{(1)} \hat{\otimes}_{i} L^{(2)}\right)^{\prime}=L^{(1) \prime} \hat{\otimes}_{i} L^{(2) \prime}$, where the hat means completion and the prime denotes the strong dual space.

The proof is given in Appendix B. In [16], it was shown that if $L^{(1)}, L^{(2)}$, and $L$ are the strong duals of reflexive Fréchet spaces, then every separately continuous bilinear map of $L^{(1)} \times L^{(2)}$ into $L$ is continuous. From Lemma 4 , it follows that if $L^{(1)}$ and $L^{(2)}$ are DFS-spaces, then an analogous statement holds for any locally convex space $L$.

Let $L^{(1)}, \ldots, L^{(n)}$ be locally convex spaces. We denote by $L^{(1)} \hat{\otimes}_{i} \ldots \hat{\otimes}_{i} L^{(n)}$ the completion of $L^{(1)} \otimes \ldots \otimes L^{(n)}$ relative to $i$-topology. If $L_{n}$ is a barrelled space, then there is the canonical identification

$$
L^{(1)} \hat{\otimes}_{i} \ldots \hat{\otimes}_{i} L^{(n)}=\left(L^{(1)} \hat{\otimes}_{i} \ldots \hat{\otimes}_{i} L^{(n-1)}\right) \hat{\otimes}_{i} L^{(n)}
$$

(to construct this isomorphism, one can use theorems III.5.2 and III.5.4 of [15]).

Lemma 5. Let $K_{1}, \ldots, K_{n}$ be nonempty closed cones in $\mathbb{R}^{k_{1}}, \ldots \mathbb{R}^{k_{n}}$ respectively and let $0 \leq \alpha<1$. Then we have the isomorphisms

$$
\begin{aligned}
& s_{\beta}^{\alpha}\left(K_{1}, \ldots, K_{n}\right)=s_{\beta}^{\alpha}\left(K_{1}, \ldots, K_{n-1}\right) \hat{\otimes}_{i} s_{\beta}^{\alpha}\left(K_{n}\right), \\
& s_{\beta}^{\prime \alpha}\left(K_{1}, \ldots, K_{n}\right)=s_{\beta}^{\prime \alpha}\left(K_{1}\right) \hat{\otimes}_{i} \ldots \hat{\otimes}_{i} s_{\beta}^{\prime \alpha}\left(K_{n}\right) .
\end{aligned}
$$

Proof. As we have already mentioned above, the spaces introduced by Definition 2 are DFS. Moreover, they are nuclear as countable inductive limits of the spaces $S_{\beta}^{\alpha}(U)$, whose nuclearity was established in [11. Since $s_{\beta}^{\alpha}\left(K_{1}, \ldots, K_{n}\right)$ is Hausdorff and complete [14, the first isomorphism follows immediately from Definition 2]and the existence of the natural identification $S_{\beta}^{\alpha}\left(U_{1} \times U_{2}\right)=S_{\beta}^{\alpha}\left(U_{1}\right) \hat{\otimes}_{i} S_{\beta}^{\alpha}\left(U_{2}\right)$ for any nonempty cones 
$U_{1}, U_{2}$, see [11], Theorem 3. The second isomorphism is obtained by induction from the first one using (10) and Lemma 4

Lemma 6. Let $G_{1}$ and $G_{2}$ be subspaces of locally convex spaces $L_{1}$ and $L_{2}$ respectively. Then the topology of equicontinuous convergence on $G_{1} \otimes G_{2}$ coincides with that induced from $L_{1} \otimes_{e} L_{2}$.

Proof. Let $j_{1,2}$ be the natural injections of $G_{1,2}$ into $L_{1,2}$ and let $j=j_{1} \otimes j_{2}$. We denote by $\mathcal{E}_{1,2}\left(\tilde{\mathcal{E}}_{1,2}\right)$ the families of equicontinuous subsets of $L_{1,2}^{\prime}$ (resp., of $\left.G_{1,2}^{\prime}\right)$. The polar sets of $\left(S_{1} \otimes S_{2}\right)^{\circ}, S_{1,2} \in \mathcal{E}_{1,2}$ form the basis of neighborhoods of the origin for $e$-topology on $L_{1} \otimes L_{2}$. Since $j_{1,2}^{\prime}\left(\mathcal{E}_{1,2}\right)=\tilde{\mathcal{E}}_{1,2}$ according to [15], Theorem IV.4.1, the sets $\left[j_{1}^{\prime}\left(S_{1}\right) \otimes j_{2}^{\prime}\left(S_{2}\right)\right]^{\circ}, S_{1,2} \in \mathcal{E}_{1,2}$, form the basis of neighborhoods of the origin for $e$-topology on $G_{1} \otimes G_{2}$. It remains to note that in view of Proposition IV.2.3a of [15] and the equality $j^{\prime}=j_{1}^{\prime} \otimes j_{2}^{\prime}$ these sets coincide with $j^{-1}\left[\left(S_{1} \otimes S_{2}\right)^{\circ}\right]$.

\section{Laplace transformation}

Definition 3. Let $\beta>1$, let $V_{1}, \ldots, V_{n}$ be nonempty open connected cones in $\mathbb{R}^{k_{1}}, \ldots, \mathbb{R}^{k_{n}}$ respectively, and let $V=V_{1} \times \ldots \times V_{n}$. The space $\mathcal{A}_{\alpha}^{\beta}\left(V_{1}, \ldots, V_{n}\right)$ with $0<\alpha<1$ (with $\alpha=0$ ) consists of analytic functions in $T^{V}=\mathbb{R}^{k_{1}} \times \ldots \times \mathbb{R}^{k_{n}}+i V$ that have, for any $\epsilon>0$ (resp., for any $R, \epsilon>0$ ), the finite norms

$$
\begin{aligned}
& \|\mathbf{v}\|_{\epsilon, V_{1}^{\prime}, \ldots, V_{n}^{\prime}}=\sup _{z \in T^{V^{\prime}}}|\mathbf{v}(z)| \prod_{j=1}^{n} \exp \left(-\epsilon\left|z_{j}\right|^{1 / \alpha}-\epsilon\left|y_{j}\right|^{-1 /(\beta-1)}\right) \\
& \left(\text { resp., }\|\mathbf{v}\|_{\epsilon, R, V_{1}^{\prime}, \ldots, V_{n}^{\prime}}=\sup _{z \in T^{V^{\prime}},\left|z_{j}\right| \leq R}|\mathbf{v}(z)| \prod_{j=1}^{n} \exp \left(-\epsilon\left|y_{j}\right|^{-1 /(\beta-1)}\right)\right), \quad y_{j}=\operatorname{Im} z_{j},
\end{aligned}
$$

where $V_{1}^{\prime}, \ldots, V_{n}^{\prime}$ are arbitrary cones compact in $V_{1}, \ldots, V_{n}$ and $V^{\prime}=V_{1}^{\prime} \times \ldots \times V_{n}^{\prime}$.

If a nondegenerate bilinear form $\langle\cdot, \cdot\rangle$ is fixed on $\mathbb{R}^{k}$, then the Fourier transform of a test function $f(x) \in S_{\alpha}^{\beta}\left(\mathbb{R}^{k}\right)$ is defined by $\hat{f}(p)=\int f(x) e^{i\langle p, x\rangle} \mathrm{d} x$. The mapping $f \rightarrow \hat{f}$ is a topological isomorphism of $S_{\alpha}^{\beta}\left(\mathbb{R}^{k}\right)$ onto $S_{\beta}^{\alpha}\left(\mathbb{R}^{k}\right)$. If $\beta>1, V$ is an open connected cone in $\mathbb{R}^{k}$, and $K=V^{*}=\{p:\langle p, y\rangle \geq 0 \forall y \in V\}$, then, as one can easily see, $e^{i\langle\cdot, z\rangle} \in s_{\beta}^{\alpha}(K)$ for any $z \in T^{V}$. The Laplace transform $\mathcal{L}_{V} u$ of a functional $u \in s_{\beta}^{\prime \alpha}(K)$ is defined by $\left(\mathcal{L}_{V} u\right)(z)=u\left(e^{i\langle\cdot, z\rangle}\right), z \in T^{V}$. As shown in [11, the Laplace operator $\mathcal{L}_{V}$ is a topological isomorphism of $s_{\beta}^{\prime \alpha}(K)$ onto $\mathcal{A}_{\alpha}^{\beta}(V)$ and hence $\mathcal{A}_{\alpha}^{\beta}(V)$ is a reflexive Fréchet space.

For $\mathbb{R}^{k}=\mathbb{R}^{k_{1}} \times \ldots \times \mathbb{R}^{k_{n}}$, we assume that $\langle p, x\rangle=\sum_{j=0}^{n}\left\langle p_{j}, x_{j}\right\rangle_{j}$, where $\langle\cdot, \cdot\rangle_{j}$ is a nondegenerate bilinear form on $\mathbb{R}^{k_{j}}$.

Lemma 7. Let $\beta>1,0 \leq \alpha<1$, and $\mathbf{v} \in \mathcal{A}_{\alpha}^{\beta}\left(V_{1}, \ldots, V_{n}\right)$, where $V_{1}, \ldots, V_{n}$ are nonempty open connected cones in $\mathbb{R}^{k_{1}}, \ldots, \mathbb{R}^{k_{n}}$ respectively. Then $\mathbf{v}\left(z_{1}, \ldots, z_{n-1}, \cdot\right) \in$ $\mathcal{A}_{\alpha}^{\beta}\left(V_{n}\right)$ for any $z_{1} \in T^{V_{1}}, \ldots, z_{n-1} \in T^{V_{n-1}}$ and $\mathbf{v}_{u}\left(z_{1}, \ldots, z_{n-1}\right)=u\left(\mathbf{v}\left(z_{1}, \ldots, z_{n-1}, \cdot\right)\right)$ belongs to $\mathcal{A}_{\alpha}^{\beta}\left(V_{1}, \ldots, V_{n-1}\right)$ for all $u \in \mathcal{A}_{\alpha}^{\prime \beta}\left(V_{n}\right)$. The mapping $u \rightarrow \mathbf{v}_{u}$ from $\mathcal{A}_{\alpha}^{\prime \beta}\left(V_{n}\right)$ into $\mathcal{A}_{\alpha}^{\beta}\left(V_{1}, \ldots, V_{n-1}\right)$ is continuous. 
Proof. We define the space $\mathrm{A}_{\alpha}^{\beta}\left(V_{1}, \ldots, V_{n}\right)$ in the same way as $\mathcal{A}_{\alpha}^{\beta}\left(V_{1}, \ldots, V_{n}\right)$ with the only difference that the elements of $\mathrm{A}_{\alpha}^{\beta}$ need not be analytic functions. $\mathcal{A}_{\alpha}^{\beta}\left(V_{1}, \ldots, V_{n}\right)$ is a closed subspace of $\mathrm{A}_{\alpha}^{\beta}\left(V_{1}, \ldots, V_{n}\right)$. Let $0<\alpha<1$, let $\epsilon>0$, and let $V_{1}^{\prime}, \ldots, V_{n}^{\prime}$ be closed subcones of $V_{1}, \ldots, V_{n}$. Set $B_{\epsilon, V_{n}^{\prime}}=\left\{u \in \mathcal{A}_{\alpha}^{\prime \beta}\left(V_{n}\right):|u(\mathbf{w})| \leq\|\mathbf{w}\|_{\epsilon, V_{n}^{\prime}} \forall \mathbf{w} \in\right.$ $\left.\mathcal{A}_{\alpha}^{\beta}\left(V_{n}\right)\right\}$. Using Definition 3, we obtain

$$
\begin{aligned}
\left|u\left(\mathbf{v}\left(z_{1}, \ldots, z_{n-1}, \cdot\right)\right)\right| \leq\left\|\mathbf{v}\left(z_{1}, \ldots, z_{n-1}, \cdot\right)\right\|_{\epsilon, V_{n}^{\prime}} & \\
& \leq\|\mathbf{v}\|_{\epsilon, V_{1}^{\prime}, \ldots, V_{n}^{\prime}} \prod_{j=1}^{n-1} \exp \left(\epsilon\left|z_{j}\right|^{1 / \alpha}+\epsilon\left|y_{j}\right|^{-1 /(\beta-1)}\right)
\end{aligned}
$$

for every $u \in B_{\epsilon, V_{n}^{\prime}}$ and every $z_{j} \in T^{V_{j}^{\prime}}, 1 \leq j \leq n-1$. Consequently, $\left\|\mathbf{v}_{u}\right\|_{\epsilon, V_{1}^{\prime}, \ldots, V_{n-1}^{\prime}} \leq$ $\|\mathbf{v}\|_{\epsilon, V_{1}^{\prime}, \ldots, V_{n}^{\prime}}$ for $u \in B_{\epsilon, V_{n}^{\prime}}$. Thus, $\mathbf{v}_{u}$ belongs to the space $\mathrm{A}_{\alpha}^{\beta}\left(V_{1}, \ldots, V_{n-1}\right)$ for any $u \in \mathcal{A}_{\alpha}^{\prime \beta}\left(V_{n}\right)$ and the image of $B_{\epsilon, V_{n}^{\prime}}$ under the mapping $u \rightarrow \mathbf{v}_{u}$ is bounded in this space. The scalar multiples of $B_{\epsilon, V_{n}^{\prime}}$ form a fundamental system of bounded subsets in the space $\mathcal{A}_{\alpha}^{\prime \beta}\left(V_{n}\right)$, which is bornologic as the strong dual of a Fréchet space, see [15], Sec. IV.6.6. Consequently, the mapping $u \rightarrow \mathbf{v}_{u}$ from $\mathcal{A}_{\alpha}^{\prime \beta}\left(V_{n}\right)$ to $\mathrm{A}_{\alpha}^{\beta}\left(V_{1}, \ldots, V_{n-1}\right)$ is continuous. Let $\delta_{z}, z \in T^{V_{n}}$, be the functional in $\mathcal{A}_{\alpha}^{\prime \beta}\left(V_{n}\right)$ which is equal to $\mathbf{w}(z)$ on the test function $\mathbf{w} \in \mathcal{A}_{\alpha}^{\beta}\left(V_{n}\right)$. Since $\mathcal{A}_{\alpha}^{\beta}\left(V_{n}\right)$ is a reflexive space, the linear span $L$ of such functionals is dense in $\mathcal{A}_{\alpha}^{\prime \beta}\left(V_{n}\right)$. It is clear that $\mathbf{v}_{u} \in \mathcal{A}_{\alpha}^{\beta}\left(V_{1}, \ldots, V_{n-1}\right)$ for any $u \in L$ and, since $\mathcal{A}_{\alpha}^{\beta}$ is closed in $\mathrm{A}_{\alpha}^{\beta}$, we have $\mathbf{v}_{u} \in \mathcal{A}_{\alpha}^{\beta}\left(V_{1}, \ldots, V_{n-1}\right)$ for any $u \in \bar{L}=\mathcal{A}_{\alpha}^{\prime \beta}\left(V_{n}\right)$. The changes in the proof for the case $\alpha=0$ are obvious. The lemma is proved.

Let $V_{1}, \ldots, V_{n}$ be nonempty open connected cones in $\mathbb{R}^{k_{1}}, \ldots, \mathbb{R}^{k_{n}}$ respectively and let $K_{j}=V_{j}^{*}, j=1, \ldots, n$. The product $\mathcal{A}_{\alpha}^{\beta}\left(V_{1}\right) \otimes_{i} \ldots \otimes_{i} \mathcal{A}_{\alpha}^{\beta}\left(V_{n}\right)$ is continuously embedded into $\mathcal{A}_{\alpha}^{\beta}\left(V_{1}, \ldots, V_{n}\right)$ by of the ordinary identification

$$
\left(\mathbf{v}_{1} \otimes \ldots \otimes \mathbf{v}_{n}\right)\left(z_{1}, \ldots, z_{n}\right)=\mathbf{v}_{1}\left(z_{1}\right) \ldots \mathbf{v}_{n}\left(z_{n}\right), \quad \mathbf{v}_{j} \in \mathcal{A}_{\alpha}^{\beta}\left(V_{j}\right) .
$$

We define the operator $\mathcal{L}_{V_{1}, \ldots, V_{n}}: s_{\beta}^{\prime \alpha}\left(K_{1}, \ldots, K_{n}\right) \rightarrow \mathcal{A}_{\alpha}^{\beta}\left(V_{1}, \ldots, V_{n}\right)$ as the continuous extension of $\mathcal{L}_{V_{1}} \otimes_{i} \ldots \otimes_{i} \mathcal{L}_{V_{n}}$ to $s_{\beta}^{\prime \alpha}\left(K_{1}, \ldots, K_{n}\right)$. By Lemma 5 and in view of the completeness of $\mathcal{A}_{\alpha}^{\beta}$, such an extension exists and is uniquely defined. For any $u \in$ $s_{\beta}^{\prime \alpha}\left(K_{1}, \ldots, K_{n}\right)$, we have

$$
\left(\mathcal{L}_{V_{1}, \ldots, V_{n}} u\right)(z)=u\left(e^{i\langle\cdot, z\rangle}\right), \quad z \in T^{V},
$$

where $V=V_{1} \times \ldots \times V_{n}$. Thus, $\mathcal{L}_{V_{1}, \ldots, V_{n}}$ is the restriction of the Laplace operator $\mathcal{L}_{V_{1} \times \ldots \times V_{n}}$ to $s_{\beta}^{\prime \alpha}\left(K_{1}, \ldots, K_{n}\right)$. To prove formula (11), it suffices to note that it holds for $u \in s_{\beta}^{\prime \alpha}\left(K_{1}\right) \otimes \ldots \otimes s_{\beta}^{\prime \alpha}\left(K_{n}\right)$ and next to make use of Lemma 5 and the continuity of both sides of the equality in $u$.

Theorem 4. Let $\beta>1,0 \leq \alpha<1$, let $V_{1}, \ldots, V_{n}$ be nonempty open connected cones in $\mathbb{R}^{k_{1}}, \ldots, \mathbb{R}^{k_{n}}$ respectively, and let $K_{j}=V_{j}^{*}, j=1, \ldots, n$. The Laplace transformation $\mathcal{L}_{V_{1}, \ldots, V_{n}}$ is a topological isomorphism of $s_{\beta}^{\prime \alpha}\left(K_{1}, \ldots, K_{n}\right)$ onto $\mathcal{A}_{\alpha}^{\beta}\left(V_{1}, \ldots, V_{n}\right)$. If $u \in$ $s_{\beta}^{\prime \alpha}\left(K_{1}, \ldots, K_{n}\right)$, then $\left(\mathcal{L}_{V_{1}, \ldots, V_{n}} u\right)(\cdot+i y)$ tends to the Fourier transform of $u$ in the strong topology of $S_{\alpha}^{\prime \beta}\left(\mathbb{R}^{k_{1}} \times \ldots \times \mathbb{R}^{k_{n}}\right)$ as $y \rightarrow 0$ inside any cone $V_{1}^{\prime} \times \ldots \times V_{n}^{\prime}$, where $V_{j}^{\prime} \Subset V_{j}, j=1, \ldots, n$. 
Proof. In [1] the statement was established for $n=1$ and it is sufficient to prove the theorem supposing it holds for the spaces over $n-1$ cones. The mapping $\mathcal{L}_{V_{1}, \ldots, V_{n}}$ is injective as the restriction of the injective operator $\mathcal{L}_{V_{1} \times \ldots \times V_{n}}$. Let $\mathbf{v} \in \mathcal{A}_{\alpha}^{\beta}\left(V_{1}, \ldots, V_{n}\right)$. We define the bilinear form $b_{\mathbf{v}}$ on $\mathcal{A}_{\alpha}^{\prime \beta}\left(V_{1}, \ldots, V_{n-1}\right) \times \mathcal{A}_{\alpha}^{\prime \beta}\left(V_{n}\right)$ by $b_{\mathbf{v}}\left(u_{1}, u_{2}\right)=u_{1}\left(\mathbf{v}_{u_{2}}\right)$. By Lemma [7] the form $b_{\mathbf{v}}$ is separately continuous. Let $T_{1}: s_{\beta}^{\alpha}\left(K_{1}, \ldots, K_{n-1}\right) \rightarrow$ $\mathcal{A}_{\alpha}^{\prime \beta}\left(V_{1}, \ldots, V_{n-1}\right)\left(T_{2}: s_{\beta}^{\alpha}\left(K_{n}\right) \rightarrow \mathcal{A}_{\alpha}^{\prime \beta}\left(V_{n}\right)\right)$ be the dual ${ }^{5}$ mapping of $\mathcal{L}_{V_{1}, \ldots, V_{n-1}}^{-1}$ (resp., of $\left.\mathcal{L}_{V_{n}}^{-1}\right)$. By Lemma [5, the separately continuous bilinear form $B_{\mathbf{v}}\left(f_{1}, f_{2}\right)=b_{\mathbf{v}}\left(T_{1} f_{1}, T_{2} f_{2}\right)$ on $s_{\beta}^{\alpha}\left(K_{1}, \ldots, K_{n-1}\right) \times s_{\beta}^{\alpha}\left(K_{n}\right)$ uniquely determines a functional $u \in s_{\beta}^{\alpha}\left(K_{1}, \ldots, K_{n}\right)$ such that $u\left(f_{1} \otimes f_{2}\right)=B_{\mathbf{v}}\left(f_{1}, f_{2}\right)$. If $z=\left(z_{1}, \ldots, z_{n}\right), \tilde{z}=\left(z_{1}, \ldots, z_{n-1}\right)$, and $z_{j} \in T^{V_{j}}$, $j=1, \ldots, n$, then

$$
u\left(e^{\langle\cdot, z\rangle}\right)=B_{\mathbf{v}}\left(e^{\langle\cdot, \tilde{z}\rangle^{\prime}}, e^{\left\langle\cdot, z_{n}\right\rangle_{n}}\right)=b_{\mathbf{v}}\left(\delta_{\tilde{z}}, \delta_{z_{n}}\right)=\mathbf{v}(z),
$$

where $\langle p, \tilde{z}\rangle^{\prime}=\sum_{j=1}^{n-1}\left\langle p_{j}, z_{j}\right\rangle_{j}, p \in \mathbb{R}^{k_{1}} \times \ldots \times \mathbb{R}^{k_{n-1}}$. Thus, $\mathbf{v}$ is the Laplace transform of $u$, i.e., the operator $\mathcal{L}_{V_{1}, \ldots, V_{n}}$ is bijective. The open mapping theorem shows that $\mathcal{L}_{V_{1}, \ldots, V_{n}}$ is a topological isomorphism. If $u \in s_{\beta}^{\prime \alpha}\left(K_{1}, \ldots, K_{n}\right)$ and $f \in S_{\alpha}^{\beta}\left(\mathbb{R}^{k_{1}+\ldots+k_{n}}\right)$, then

$$
\int\left(\mathcal{L}_{V_{1}, \ldots, V_{n}} u\right)(x+i y) f(x) \mathrm{d} x=u\left(e^{-\langle\cdot, y\rangle} \hat{f}\right), \quad y \in V_{1} \times \ldots \times V_{n} .
$$

Indeed, the formula holds for $n=1$, see [1], and $\mathcal{L}_{V_{1}, \ldots, V_{n}} u$ coincides with $\mathcal{L}_{V_{1} \times \ldots \times V_{n}} u$. The direct check shows that $e^{-\langle\cdot, y\rangle} \hat{f} \rightarrow \hat{f}$ in the topology of $s_{\beta}^{\alpha}\left(K_{1}, \ldots, K_{n}\right)$ as $y \rightarrow 0$ inside $V_{1}^{\prime} \times \ldots \times V_{n}^{\prime}$. Therefore, to prove the last statement of the theorem, it suffices to apply (12) and to take into account that in the Montel space $S_{\alpha}^{\prime \beta}$, weak convergence and strong convergence are equivalent.

\section{Euclidean reconstruction theorem}

From now on, the Lorentz product $p^{0} x^{0}-p^{1} x^{1}-\ldots-p^{d-1} x^{d-1}$ of $p, x \in \mathbb{R}^{d}$ will be denoted by $p x$.

All requirements of the Wightman formalism except for the spectral condition are formulated in the usual way for the fields of the class $S_{\alpha}^{\prime \beta}, 0 \leq \alpha<1, \beta>1$ (under the condition $\beta>1$, the local commutativity is formulated as usual). As we have already noted in Introduction, the spectral condition in standard form (11) is inapplicable in this case because of the lack of test functions of compact support in $p$-space. To obtain an appropriate generalization of the spectral condition, one can use the notion of strong carrier cone introduced in Section 2. As a result, we come to the following set of axioms for the Wightman functions:

W1 (Growth and singularity) $w_{n} \in S_{\alpha}^{\prime \beta}\left(\mathbb{R}^{d n}\right) 0 \leq \alpha<1, \beta>1$;

W2 (Relativistic invariance) $w_{n}\left(\Lambda x_{1}+a, \ldots, \Lambda x_{n}+a\right)=w_{n}\left(x_{1}, \ldots, x_{n}\right)$ for any proper Lorentz transformation $\Lambda$ and vector $a \in \mathbb{R}^{d}$;

\footnotetext{
${ }^{5}$ Since $s_{\beta}^{\alpha}$ are reflexive spaces, we identify $s_{\beta}^{\prime \prime \alpha}$ with $s_{\beta}^{\alpha}$.
} 
W3 (Generalized spectral condition) $\overline{\mathbb{V}}_{+}^{n}$ is a strong carrier cone of $\hat{W}_{n}$, i.e., $\hat{W}_{n} \in$ $s_{\beta}^{\prime \alpha}\left(\overline{\mathbb{V}}_{+}, \ldots, \overline{\mathbb{V}}_{+}\right)$

W4 (Locality) $w_{n}\left(x_{1}, \ldots, x_{j}, x_{j+1}, x_{n}\right)-w_{n}\left(x_{1}, \ldots, x_{j+1}, x_{j}, \ldots, x_{n}\right)=0$ if $x_{j}-x_{j+1}$ is space-like.

We do not impose the positivity condition on $w_{n}$, which corresponds to the case of an indefinite metric in the state space. Besides, we do not require the fulfillment of the cluster property which is not equivalent to the uniqueness of the vacuum in indefinite metric theories, see 4 .

It should be noted that in the indefinite metric case, theory is not determined uniquely by its Wightman functions and to obtain its complete operator realization, it is necessary to specify, in addition to the sequence $w_{n}$, the Hilbert majorant of the indefinite metric which determines the convergence in the state space 4]. For simplicity, we restrict our consideration to Wightman functions and do not touch here more subtle questions concerning the construction of the Hilbert majorant.

Using Theorem 4 and condition W3, we conclude ${ }^{6}$ that $W_{n}(\xi)$ is the boundary value of the function $\mathbf{W}_{n}(\zeta)=(2 \pi)^{-d n} \mathcal{L}_{\mathbb{V}_{-}, \ldots, \mathbb{V}_{-}} \hat{W}_{n}$ holomorphic in the past tube $\mathbb{R}^{d n}+i \mathbb{V}_{-}^{n}$. Correspondingly, $w_{n}$ is the boundary value of the function $\mathbf{w}_{n}\left(z_{1}, \ldots, z_{n}\right)=\mathbf{W}_{n-1}\left(z_{1}-\right.$ $\left.z_{2}, \ldots, z_{n-1}-z_{n}\right)$ holomorphic in the domain $\left\{z: z_{j}-z_{j+1} \in \mathbb{R}^{d}+i \mathbb{V}_{-}\right\}$. Standard analysis [17] based on the relativistic invariance and locality shows that $\mathbf{w}_{n}$ can be continued analytically to the extended domain $O_{n}^{e x t}$ which is invariant under the complex Lorentz transformations and the permutations of arguments. For $x=\left(x_{1}, \ldots, x_{n}\right) \in$ $\mathbb{R}^{d n}$, we set $\iota x=\left(\iota x_{1}, \ldots, \iota x_{n}\right)$, where $\iota x_{j}=\left(i x_{j}^{0}, x_{j}^{1}, \ldots, x_{j}^{d-1}\right)$. Then $\iota x \in O_{n}^{e x t}$ if and only if $x \in \mathbb{R}_{\neq}^{d n}=\left\{x \in \mathbb{R}^{d n}: x_{i} \neq x_{j}, 1 \leq i<j \leq n\right\}$, see [2, [5]. The Schwinger functions $s_{n}$ are defined by the relation $s_{n}(x)=\mathbf{w}_{n}(\iota x), x \in \mathbb{R}_{\neq}^{d n}$. In the same way as in the ordinary theory [2, 5], we establish that $s_{n}$ are rotationally invariant and symmetric with respect to the permutations of arguments. Let $S_{n}(\xi)$ be the Schwinger function $s_{n+1}$ expressed in terms of the difference variables $\xi_{j}=x_{j}-x_{j+1}$, and let $\mathbb{R}_{-}^{d n}=\left\{x \in \mathbb{R}^{d n}: x_{j}^{0}<0, j=1, \ldots, n\right\}$. If $\xi \in \mathbb{R}_{-}^{d n}$, then $\iota \xi$ lies in the past tube and by Theorem 4 , the function $S_{n}(\xi)=\mathbf{W}_{n}(\iota \xi)$ satisfies, for $0<\alpha<1$ (for $\alpha=0$ ), the bound

$$
\begin{array}{ll}
\left|S_{n}(\xi)\right| \leq C_{\epsilon} \exp \left[\epsilon|\xi|^{1 / \alpha}+\epsilon\left(\min _{1 \leq j \leq n}\left|\xi_{j}^{0}\right|\right)^{-1 /(\beta-1)}\right], & \xi \in \mathbb{R}_{-}^{d n}, \\
\text { (resp., }\left|S_{n}(\xi)\right| \leq C_{\epsilon, R} \exp \left[\epsilon\left(\min _{1 \leq j \leq n}\left|\xi_{j}^{0}\right|\right)^{-1 /(\beta-1)}\right], & \left.\xi \in \mathbb{R}_{-}^{d n},|\xi| \leq R\right)
\end{array}
$$

for any $\epsilon>0$ (resp., for any $\epsilon, R>0$ ). As shown in [5] (see the proof of Theorem 9.30), for any $x \in \mathbb{R}_{\neq}^{d n}$ there exist a rotation $T$ and a permutation $\pi$ of the set $[1 . . n]$ such that

$$
\min _{1 \leq j \leq n-1}\left[\left(T x_{\pi(j+1)}\right)^{0}-\left(T x_{\pi(j)}\right)^{0}\right] \geq c \min _{j \neq k}\left|x_{j}-x_{k}\right|,
$$

where $c$ is a positive constant depending only on $n$. In view of the invariance of the Schwinger functions under rotations and permutations of arguments, (13) and (14)

\footnotetext{
${ }^{6}$ Here and subsequently, applying Theorem 4 we set $\langle p, x\rangle=-\sum_{j=1}^{n} p_{j} x_{j}, p, x \in \mathbb{R}^{d n}$.
} 
imply the inequality

$$
\begin{array}{ll}
\left|s_{n}(x)\right| \leq C_{\epsilon} \exp \left[\epsilon|x|^{1 / \alpha}+\epsilon\left(\min _{j \neq k}\left|x_{j}-x_{k}\right|\right)^{-1 /(\beta-1)}\right], & x \in \mathbb{R}_{\neq}^{d n}, \\
(\operatorname{resp} ., & \left.\left|s_{n}(x)\right| \leq C_{\epsilon, R} \exp \left[\epsilon\left(\min _{j \neq k}\left|x_{j}-x_{k}\right|\right)^{-1 /(\beta-1)}\right], \quad x \in \mathbb{R}_{\neq}^{d n},|x| \leq R\right) .
\end{array}
$$

for any $\epsilon>0$ (resp., for any $\epsilon, R>0$ ). The obtained estimates allow interpreting $s_{n}$ as generalized functions defined under smearing with suitable test functions. The relevant test function spaces are introduced by the following definition.

Definition 4. Let $\alpha \geq 0, \beta>1$ and let $O$ be an open set in $\mathbb{R}^{k}$. We denote by $\Sigma_{\alpha}^{\beta}(O)$ the subspace of $S_{\alpha}^{\beta}\left(\mathbb{R}^{k}\right)$ consisting of those functions that are identically zero on the complement $\complement O$ of $O$ together with all their derivatives.

$\Sigma_{\alpha}^{\beta}(O)$ is a closed subspace of $S_{\alpha}^{\beta}\left(\mathbb{R}^{k}\right)$. Therefore, by Theorem $7^{\prime}$ of [14, we have $\Sigma_{\alpha}^{\beta}(O)=\underline{\lim }_{A, B>0} \Sigma_{\alpha, A}^{\beta, B}(O)$, where $\Sigma_{\alpha, A}^{\beta, B}(O)$ is the Banach space consisting of the functions $f \in \Sigma_{\alpha}^{\beta}(O)$ such that \|\|$f \|_{B, A}<\infty$ (see formula (3i) ).

Lemma 8. Let $O$ be an open set in $\mathbb{R}^{k}$. If $\alpha>0$ (if $\alpha=0$ ), then for any $A, B>0$ there is $A^{\prime}>0$ such that for all $x \in O$ and $f \in \Sigma_{\alpha, A}^{\beta, B}(O)$ the inequality

$$
\begin{aligned}
& |f(x)| \leq C|||f| \|_{B, A} \exp \left[-A^{\prime}|x|^{1 / \alpha}-A^{\prime}\left(\delta_{\mathrm{CO}}(x)\right)^{-1 /(\beta-1)}\right] \\
& \left(\text { resp., }|f(x)| \leq C \mid\|f\|_{B, A} \exp \left[-A^{\prime}\left(\delta_{\mathrm{CO}}(x)\right)^{-1 /(\beta-1)}\right] \text { and } f(x)=0 \text { for }|x| \geq A\right),
\end{aligned}
$$

is valid, where $\delta_{\mathrm{Co}}(x)$ is the distance from $x$ to $\mathrm{CO}$.

Proof. Let $f \in \Sigma_{\alpha, A}^{\beta, B}(O), x \in O$ and $x_{0}$ be a point in $\complement O$ such that $\left|x-x_{0}\right|=\delta_{\mathrm{CO}}(x)$. By Taylor's formula, for every $m \in \mathbb{N}=0,1, \ldots$ we have $f(x)=\sum_{|\lambda|=m} \partial^{\lambda} f\left(x_{0}+\right.$ th) $h^{\lambda} / \lambda$ !, where $0<t<1, h=x-x_{0}$, and the standard multi-index notation is used. From (3) it follows that $\left|\partial^{\lambda} f(x)\right| \leq\left.|| f\left|\|_{B, A} B^{|\lambda|}\right| \lambda\right|^{\beta|\lambda|}$. Since $\left|h^{\lambda}\right| \leq|h|^{|\lambda|}$, we get $|f(x)| \leq\left\||| f\left|\left\|_{B, A}(B|h|)^{m} m^{\beta m} \sum_{|\lambda|=m} 1 / \lambda !=\right\|\right| f \mid\right\|_{B, A}(B|h| k)^{m} m^{\beta m} / m$ ! and using the inequality $m ! \geq(m / e)^{m}$, we find that $|f(x)| \leq\||f|\|_{B, A} \inf _{m \in \mathbb{N}}(B|h| k e)^{m} m^{(\beta-1) m}$. As shown in [13, Sec. IV.2, $\inf _{m} \xi^{-m} m^{\alpha m} \leq \exp \left(-\frac{\alpha}{e} \xi^{1 / \alpha}+\alpha e / 2\right)$ for any $\xi, \alpha>0$. Replacing $\alpha$ and $\xi$ with $\beta-1$ and $1 / B|h| k e$ respectively, we obtain

$$
|f(x)| \leq C_{1}|||f| \|_{B, A} \exp \left(-\frac{(\beta-1)}{e}\left(B k e \delta_{\mathrm{CO}}(x)\right)^{-\frac{1}{\beta-1}}\right) .
$$

On the other hand, by (3) we have $|f(x)| \leq\||f|\|_{B, A} \inf _{m \in \mathbb{N}}(A /|x|)^{m} m^{\alpha m}$. For $\alpha=0$, this implies that $f(x)=0$ for $|x|>A$. If $\alpha>0$, then an analogous estimation of the infimum shows that $|f(x)| \leq C_{2}|||f| \|_{B, A} \exp \left(-\frac{\alpha}{e}(|x| / A)^{1 / \alpha}\right)$. Multiplying the last estimate and inequality (16) and taking the square root of the left- and right-hand sides, we arrive at the statement of the lemma.

Since $\delta_{\mathbb{C R}_{\neq}^{d n}}(x) \leq \min _{j \neq k}\left|x_{j}-x_{k}\right|$ for $x \in \mathbb{R}_{\neq}^{d n}$, Lemma 8 and the estimate (15) imply that $s_{n} \in \Sigma_{\alpha}^{\prime \beta}\left(\mathbb{R}_{\neq}^{d n}\right)$. Analogously, from (13) it follows that $S_{n} \in \Sigma_{\alpha}^{\prime \beta}\left(\mathbb{R}_{-}^{d n}\right)$.

For $\mathbf{v} \in \mathcal{A}_{\alpha}^{\beta}\left(\mathbb{V}_{-}, \ldots, \mathbb{V}_{-}\right)$, we set $l_{\mathbf{v}}(f)=(2 \pi)^{-d n} \int_{\mathbb{R}^{d n}} \mathbf{v}(\iota x) f(x) \mathrm{d} x, f \in \Sigma_{\alpha}^{\beta}\left(\mathbb{R}_{-}^{d n}\right)$. By Lemma 8 , the mapping $\mathbf{v} \rightarrow l_{\mathbf{v}}$ from $\mathcal{A}_{\alpha}^{\beta}\left(\mathbb{V}_{-}, \ldots, \mathbb{V}_{-}\right)$into $\Sigma_{\alpha}^{\prime \beta}\left(\mathbb{R}_{-}^{d n}\right)$ is continuous. 
Consequently, for every fixed $f \in \Sigma_{\alpha}^{\beta}\left(\mathbb{R}_{-}^{d n}\right)$ the functional $u \rightarrow l_{\mathcal{L}_{\mathbb{V}_{-}, \ldots, V_{-} u}}(f)$ is continuous on $s_{\beta}^{\prime \alpha}\left(\overline{\mathbb{V}}_{+}, \ldots, \overline{\mathbb{V}}_{+}\right)$and because of the reflexivity of the latter space there is an element $\tilde{f} \in s_{\beta}^{\alpha}\left(\overline{\mathbb{V}}_{+}, \ldots, \overline{\mathbb{V}}_{+}\right)$such that

$$
(2 \pi)^{-d n} \int_{\mathbb{R}_{-}^{d n}}\left(\mathcal{L}_{\mathbb{V}_{-}, \ldots, V_{-}} u\right)(\iota \xi) f(\xi) \mathrm{d} \xi=u(\check{f}), \quad u \in s_{\beta}^{\prime \alpha}\left(\overline{\mathbb{V}}_{+}, \ldots, \overline{\mathbb{V}}_{+}\right) .
$$

Taking $u=\delta_{p}$ (the value of $\delta_{p}$ on a test function $g$ is equal to $g(p)$ ), we find that

$$
\check{f}(p)=(2 \pi)^{-d n} \int_{\mathbb{R}_{-}^{d n}} f(\xi) \exp \left[\sum_{j=1}^{n}\left(p_{j}^{0} \xi_{j}^{0}-i p_{j}^{1} \xi_{j}^{1}-\ldots-i p_{j}^{d-1} \xi_{j}^{d-1}\right)\right] \mathrm{d} \xi .
$$

The mapping $f \rightarrow \check{f}$ from $\Sigma_{\alpha}^{\beta}\left(\mathbb{R}_{-}^{d n}\right)$ to $s_{\beta}^{\alpha}\left(\overline{\mathbb{V}}_{+}, \ldots, \overline{\mathbb{V}}_{+}\right)$has the continuous injective mapping $u \rightarrow l_{\mathcal{L}_{\mathbb{V}_{-}, \ldots, V_{-} u}}$ as its dual. As a consequence, it is a continuous mapping with dense image.

Lemma 9. The mapping $f \rightarrow \check{f}$ defined by (18) is a continuous dense embedding of $\Sigma_{\alpha}^{\beta}\left(\mathbb{R}_{-}^{d n}\right)$ into $S_{\beta}^{\alpha}\left(\mathbb{R}_{+}^{d n}\right)$, where $\mathbb{R}_{+}^{d n}=-\mathbb{R}_{-}^{d n}$.

To prove the lemma, we need the following auxiliary statement.

Lemma 10. Let $V_{1}$ and $V_{2}$ be nonempty open convex cones in $\mathbb{R}^{k_{1}}$ and $\mathbb{R}^{k_{2}}$ respectively. Then $\Sigma_{\alpha}^{\beta}\left(V_{1} \times V_{2}\right)=\Sigma_{\alpha}^{\beta}\left(V_{1}\right) \hat{\otimes}_{i} \Sigma_{\alpha}^{\beta}\left(V_{2}\right)$.

Proof. Applying Lemma 4 to the nuclear DFS-spaces $\Sigma_{\alpha}^{\beta}\left(V_{1,2}\right)$, we obtain $\Sigma_{\alpha}^{\beta}\left(V_{1}\right) \otimes_{i}$ $\Sigma_{\alpha}^{\beta}\left(V_{2}\right)=\Sigma_{\alpha}^{\beta}\left(V_{1}\right) \otimes_{e} \Sigma_{\alpha}^{\beta}\left(V_{2}\right)$ and by Lemma [6] it suffices to show that the tensor product $\Sigma_{\alpha}^{\beta}\left(V_{1}\right) \otimes \Sigma_{\alpha}^{\beta}\left(V_{2}\right)$ is dense in $\Sigma_{\alpha}^{\beta}\left(V_{1} \times V_{2}\right)$. In other words, we have to demonstrate that if a functional $u \in S_{\alpha}^{\prime \beta}\left(\mathbb{R}^{k_{1}+k_{2}}\right)$ vanishes on $\Sigma_{\alpha}^{\beta}\left(V_{1}\right) \otimes \Sigma_{\alpha}^{\beta}\left(V_{2}\right)$, then it also vanishes on $\Sigma_{\alpha}^{\beta}\left(V_{1} \times V_{2}\right)$. To this end, we take $\psi_{1,2} \in \Sigma_{\alpha}^{\beta}\left(-V_{1,2}\right)$ such that $\int_{\mathbb{R}^{k_{1,2}}} \psi_{1,2} \mathrm{~d} x=1$ and set $\Psi_{\varepsilon}\left(x_{1}, x_{2}\right)=\varepsilon^{-k_{1}-k_{2}} \psi_{1}\left(x_{1} / \varepsilon\right) \psi_{2}\left(x_{2} / \varepsilon\right)$. If $x \in \bar{V}_{1} \times \bar{V}_{2}$, then $\Psi_{\varepsilon}(x-\cdot) \in \Sigma_{\alpha}^{\beta}\left(V_{1}\right) \otimes \Sigma_{\alpha}^{\beta}\left(V_{2}\right)$ and, consequently, $\left(u * \Psi_{\varepsilon}\right)(x)=0$. Hence, for $f \in \Sigma_{\alpha}^{\beta}\left(V_{1} \times V_{2}\right)$, we have $u(f)=$ $\lim _{\varepsilon \rightarrow 0} \int_{\bar{V}_{1} \times \bar{V}_{2}}\left(u * \Psi_{\varepsilon}\right)(x) f(x) \mathrm{d} x=0$. The lemma is proved.

Proof of Lemma [9. If $\check{f}=0$, then setting $u=\delta_{\iota p}$ in (17), we see that the Fourier transform of $f$ vanishes and hence $f=0$. Thus, the mapping $f \rightarrow \check{f}$ is injective. For $f \in \Sigma_{\alpha}^{\beta}\left(\mathbb{R}_{-}\right)$, we set $\tilde{f}(p)=(2 \pi)^{-1} \int_{\mathbb{R}_{-}} f(\xi) e^{\xi p} \mathrm{~d} \xi$. In the same way as above (see the paragraph preceding the formulation of Lemma 9 ), we establish that $\tilde{f} \in s_{\beta}^{\alpha}\left(\overline{\mathbb{R}}_{+}\right)=$ $S_{\beta}^{\alpha}\left(\mathbb{R}_{+}\right)$and that the mapping $P$ taking $f$ to $\tilde{f}$ is a continuous dense embedding of $\Sigma_{\alpha}^{\beta}\left(\mathbb{R}_{-}\right)$into $S_{\beta}^{\alpha}\left(\mathbb{R}_{+}\right)$. By Lemma 10, we have $\Sigma_{\alpha}^{\beta}\left(\mathbb{R}_{-}^{d}\right)=\Sigma_{\alpha}^{\beta}\left(\mathbb{R}_{-}\right) \hat{\otimes}_{i} \Sigma_{\alpha}^{\beta}\left(\mathbb{R}^{d-1}\right)$ and Theorem 3 of [11] ensures that $S_{\beta}^{\alpha}\left(\mathbb{R}_{+}^{d}\right)=S_{\beta}^{\alpha}\left(\mathbb{R}_{+}\right) \hat{\otimes}_{i} S_{\beta}^{\alpha}\left(\mathbb{R}^{d-1}\right)$. Let $L_{1}=P \hat{\otimes}_{i} \mathcal{F}$, where $\mathcal{F}$ is the (inverse) Fourier transformation on $\mathbb{R}^{d-1}$ :

$$
(\mathcal{F} f)\left(p^{1}, \ldots, p^{d-1}\right)=(2 \pi)^{-(d-1)} \int_{\mathbb{R}^{d-1}} f(\xi) e^{-i \xi^{1} p^{1}-\ldots-i \xi^{d-1} p^{d-1}} \mathrm{~d} \xi .
$$

Obviously, $L_{1}$ is a continuous operator from $\Sigma_{\alpha}^{\beta}\left(\mathbb{R}_{-}^{d}\right)$ to $S_{\beta}^{\alpha}\left(\mathbb{R}_{+}^{d}\right)$ with a dense image. Besides, $\left(L_{1} f\right)(p)=\check{f}(p)$ for all $f \in \Sigma_{\alpha}^{\beta}\left(\mathbb{R}_{-}^{d}\right)$. Indeed, this equality holds for $f \in$ $\Sigma_{\alpha}^{\beta}\left(\mathbb{R}_{-}\right) \otimes \Sigma_{\alpha}^{\beta}\left(\mathbb{R}^{d-1}\right)$, and since both sides of the equality are continuous in $f$, it is 
valid everywhere on $\Sigma_{\alpha}^{\beta}\left(\mathbb{R}_{-}^{d}\right)$. Thus, the lemma is proved for $n=1$. For $n>1$, we make use of the representations $\Sigma_{\alpha}^{\beta}\left(\mathbb{R}_{-}^{d n}\right)=\Sigma_{\alpha}^{\beta}\left(\mathbb{R}_{-}^{d}\right) \hat{\otimes}_{i} \ldots \hat{\otimes}_{i} \Sigma_{\alpha}^{\beta}\left(\mathbb{R}_{-}^{d}\right)$ and $S_{\beta}^{\alpha}\left(\mathbb{R}_{+}^{d n}\right)=$ $S_{\beta}^{\alpha}\left(\mathbb{R}_{+}^{d}\right) \hat{\otimes}_{i} \ldots \hat{\otimes}_{i} S_{\beta}^{\alpha}\left(\mathbb{R}_{+}^{d}\right)$ which follow by induction from (10), Lemma 10] and Theorem 3 of [1]. Setting $L_{n}=L_{1} \hat{\otimes}_{i} \ldots \hat{\otimes}_{i} L_{1}$ and arguing as above, we make sure that $L_{n}$ is a continuous operator from $\Sigma_{\alpha}^{\beta}\left(\mathbb{R}_{-}^{d n}\right)$ to $S_{\beta}^{\alpha}\left(\mathbb{R}_{+}^{d n}\right)$ with dense image and such that $\left(L_{n} f\right)(p)=\check{f}(p)$. The lemma is proved.

Substituting $u=\hat{W}_{n}$ in (17) yields

$$
\int_{\mathbb{R}_{-}^{d n}} S_{n}(x) f(x) \mathrm{d} x=\hat{W}_{n}(\check{f}), \quad f \in \Sigma_{\alpha}^{\beta}\left(\mathbb{R}_{-}^{d n}\right) .
$$

By condition (W3), there is a continuous seminorm $P$ on $S_{\beta}^{\alpha}\left(\mathbb{R}_{+}^{d n}\right)$ such that $\left|\hat{W}_{n}(f)\right| \leq$ $P(f)$ for every test function in $S_{\beta}^{\alpha}\left(\mathbb{R}_{+}^{d n}\right)$. By Lemma 9 and equality (19), it hence follows that $\left|S_{n}(f)\right| \leq P(\check{f}), f \in \Sigma_{\alpha}^{\beta}\left(\mathbb{R}_{-}^{d n}\right)$.

Summarizing the above discussion, we obtain the following set of conditions on the Schwinger functions:

S1 (Growth and singularity) $s_{n} \in \Sigma_{\alpha}^{\prime \beta}\left(\mathbb{R}_{\neq}^{d n}\right)$;

S2 (Euclidean invariance) $s_{n}\left(T x_{1}+a, \ldots, T x_{n}+a\right)=s_{n}\left(x_{1}, \ldots, x_{n}\right)$ for any rotation $T$ and any $a \in \mathbb{R}^{d}$;

S3 (Laplace transform condition) There is a continuous seminorm $P$ on $S_{\beta}^{\alpha}\left(\mathbb{R}_{+}^{d n}\right)$ such that for every $f \in \Sigma_{\alpha}^{\beta}\left(\mathbb{R}_{-}^{d n}\right)$ the inequality $\left|S_{n}(f)\right| \leq P(\check{f})$ holds, where $\check{f}$ is the function defined by formula (18);

S4 (Symmetry) $s_{n}\left(x_{\pi(1)}, \ldots, x_{\pi(n)}\right)=s_{n}\left(x_{1}, \ldots, x_{n}\right)$ for all permutations $\pi$ of the indices.

We now can formulate the main result.

Theorem 5. For a given sequence of Wightman functions $w_{n}$ satisfying W1-W4, the corresponding sequence of the Schwinger functions $s_{n}$ satisfies $\mathrm{S} 1-\mathrm{S} 4$. Conversely, generalized functions satisfying S1-S4 are the Schwinger functions corresponding to a uniquely determined sequence of Wightman functions satisfying W1-W4.

Proof. The construction of the Schwinger functions corresponding to given Wightman functions and the derivation of the properties S1-S4 are given above and we we only need to prove the converse statement. Let the sequence $s_{n}$ satisfy S1-S4 and let $L$ denote the image of $\mathbb{R}_{-}^{d n}$ under the mapping $f \rightarrow \check{f}$. By S3, the linear functional $\check{f} \rightarrow S_{n}(f)$ defined on $L$ is continuous in the topology of $S_{\beta}^{\alpha}\left(\mathbb{R}_{+}^{d n}\right)$ and in view of Lemma 9 there is a uniquely determined generalized function $\hat{W}_{n} \in S_{\beta}^{\prime \alpha}\left(\mathbb{R}_{+}^{d n}\right)$ such that $\hat{W}_{n}(\check{f})=S_{n}(f), f \in \Sigma_{\alpha}^{\beta}\left(\mathbb{R}_{-}^{d n}\right)$. The invariance of $\hat{W}_{n}$ under spatial rotations follows immediately from $\mathrm{S} 2$. To prove the invariance of $\hat{W}_{n}$ under pure Lorentz transformations, it suffices to show that $X_{0 l} \hat{W}_{n}=0$, where $l=1,2,3, X_{0 l}=\sum_{k=0}^{n}\left(p_{k}^{0} \partial / \partial p_{k}^{l}+p_{k}^{l} \partial / \partial p_{k}^{0}\right)$ are the infinitesimal generators of boosts. Let $Y_{0 l}=\sum_{k=0}^{n}\left(\xi_{k}^{0} \partial / \partial \xi_{k}^{l}-\xi_{k}^{l} \partial / \partial \xi_{k}^{0}\right)$ be the 
infinitesimal generators of Euclidean rotations. It is easy to see that $Y_{0 l} f$ is taken to $X_{0 l} \check{f}$ by the mapping $f \rightarrow \check{f}$ and hence $X_{0 l} \hat{W}_{n}$ vanishes on $L$ :

$$
\left(X_{0 l} \hat{W}_{n}\right)(\check{f})=-W_{n}\left(X_{0 l} \check{f}\right)=-S_{n}\left(Y_{0 l} f\right)=\left(Y_{0 l} S_{n}\right)(f)=0, \quad f \in \Sigma_{\alpha}^{\beta}\left(\mathbb{R}_{-}^{d n}\right) .
$$

Using Lemma 9 and the continuity of $\hat{W}_{n}$, we conclude that $X_{0 l} \hat{W}_{n}=0$. By the proven Lorentz invariance, $\hat{W}_{n}$ belongs not only to $S_{\beta}^{\prime \alpha}\left(\mathbb{R}_{+}^{d n}\right)$, but also to every space $S_{\beta}^{\prime \alpha}\left(\left(\Lambda \mathbb{R}_{+}^{d}\right)^{n}\right)$, where $\Lambda$ is a proper Lorentz transformation, and, moreover, to every space $s_{\beta}^{\prime \alpha}\left(\Lambda \overline{\mathbb{R}}_{+}^{d}, \ldots, \Lambda \overline{\mathbb{R}}_{+}^{d}\right)$. Applying Theorem 2 and using the equality $\cap_{\Lambda} \Lambda \overline{\mathbb{R}}_{+}^{d}=\overline{\mathbb{V}}_{+}$, we conclude that $\overline{\mathbb{V}}_{+}^{n}$ is a strong carrier cone of $\hat{W}_{n}$. We now define the Wightman functions $w_{n}$ by formula (21) and the second relation in (11). Obviously, $w_{n}$ satisfy conditions W1, W2, and W3. Substituting $u=\hat{W}_{n}$ in (17) shows that $s_{n}$ are indeed the Schwinger functions corresponding to $w_{n}$. The symmetry of $s_{n}$ implies the symmetry of the Wightman functions $w_{n}$ in their ordinary analyticity domain, whence property W4 is derived by the standard arguments [17. The theorem is proved.

\section{$6 \quad$ Wick power series}

In this section, we show that the generalized spectral condition formulated in the previous section is satisfied for the simplest examples of quantum fields with highly singular infrared behavior, namely, for the fields representable by infinite series in the Wick powers of an indefinite metric free field $\phi$, i.e., by series of the form

$$
\sum_{k=0}^{\infty} d_{k}: \phi^{k}:(x) \text {. }
$$

We assume that $\phi$ is a tempered operator-valued distribution acting in a Hilbert-Krein state space $\mathcal{H}$ (see [4] for the role of this condition). This means that $\mathcal{H}$ is endowed, in addition to an indefinite metric $\langle\cdot, \cdot\rangle$, by an auxiliary positive scalar product $(\cdot, \cdot)$ connected with $\langle\cdot, \cdot\rangle$ by the relation $\langle\Phi, \Psi\rangle=(\Phi, \theta \Psi)$, where $\Phi, \Psi \in \mathcal{H}$ and $\theta$ is a bounded self-adjoint operator such that $\theta^{2}=1$. The scalar product $(\cdot, \cdot)$ determines a distribution $w_{\mathrm{maj}}$, which is called the majorant of the two-point vacuum average $w\left(x-x^{\prime}\right)=\left\langle\Psi_{0}, \phi(x) \phi\left(x^{\prime}\right) \Psi_{0}\right\rangle$, by the relation

$$
\left(\phi(f) \Psi_{0}, \phi(g) \Psi_{0}\right)=\int w_{\text {maj }}\left(x, x^{\prime}\right) \bar{f}(x) g\left(x^{\prime}\right) \mathrm{d} x \mathrm{~d} x^{\prime}
$$

where $\Psi_{0}$ is the vacuum and $f, g$ are test functions in the Schwartz space $S\left(\mathbb{R}^{d}\right)$. The the Krein structure implies [18 that $w_{\text {maj }}\left(x, x^{\prime}\right)$ is the boundary value of a function $\mathbf{w}_{\text {maj }}\left(z, z^{\prime}\right)$ holomorphic in the tubular domain $\left\{\left(z, z^{\prime}\right) \in \mathbb{C}^{2 d}: y=\operatorname{Im} z \in \mathbb{V}_{-}, y^{\prime}=\right.$ $\left.\operatorname{Im} z^{\prime} \in \mathbb{V}_{+}\right\}$. As in [19, we find it convenient to characterize the infrared and ultraviolet behavior of the majorant by a pair of monotone nonnegative functions $w_{I R}$ and $w_{U V}$ 
increasing as their arguments tend to infinity and to zero, respectively, and satisfying the estimate

$$
\left|\mathbf{w}_{\text {maj }}\left(z, z^{\prime}\right)\right| \leq C\left(1+w_{I R}\left(|z|+\left|z^{\prime}\right|\right)+w_{U V}\left(|y|+\left|y^{\prime}\right|\right)\right), \quad\left(y, y^{\prime}\right) \in V \times V^{\prime},
$$

for any compact subcones $V$ and $V^{\prime}$ of $\mathbb{V}_{-}$and $\mathbb{V}_{+}$(with constant $C$ depending on $V$ and $V^{\prime}$ ). Formula (21) also allows to estimate the analytic two-point Wightman function $\mathbf{w}(z)$ because

$$
\left|\mathbf{w}\left(x-x^{\prime}-2 i y\right)\right|^{2} \leq\left|\mathbf{w}_{\text {maj }}(x-i y, x+i y)\right|\left|\mathbf{w}_{\text {maj }}\left(x^{\prime}-i y, x^{\prime}+i y\right)\right|
$$

for all $y \in \mathbb{V}_{+}$. Indeed, as $\theta^{2}=1$, we have

$$
\left|\left\langle\phi(f) \Psi_{0}, \phi(g) \Psi_{0}\right\rangle\right| \leq\left\|\phi(f) \Psi_{0}\right\|\left\|\phi(g) \Psi_{0}\right\| .
$$

Taking $f(\xi)=(\nu / \sqrt{\pi})^{\mathrm{d}} e^{-\nu^{2}(\xi-x-i y)^{2}}$ and $g(\xi)=(\nu / \sqrt{\pi})^{\mathrm{d}} e^{-\nu^{2}\left(\xi-x^{\prime}-i y\right)^{2}}$ and writing the left- and right-hand sides in this inequality as integrals over a plane in the analyticity domain and passing to the limit as $\nu \rightarrow \infty$, we immediately obtain (22). Choosing $V^{\prime}=-V$ in (21) and substituting (21) in (22) yield

$$
|\mathbf{w}(\zeta)| \leq C\left(1+w_{I R}(2|\zeta|)+w_{U V}(|\eta|)\right), \quad \eta=\operatorname{Im} \zeta \in V
$$

for any compact subcone $V$ of $\mathbb{V}_{\text {- with }} C$ depending on $V$.

The following criterion allows finding the adequate test function space on which the series (20) is convergent.

Theorem 6. Let $\phi$ be a free field acting in a Hilbert-Krein space $\mathcal{H}$, and let the positive majorant of its two-point Wightman function satisfy the inequality (21) with monotonic $w_{I R}$ and $w_{U V}$. Let the coefficients $d_{k}$ satisfy the condition

$$
\left|d_{k} d_{l}\right| \leq A h^{k+l}\left|d_{k+l}\right|
$$

with some $A, h>0$. Then the series (20) is well defined as an operator-valued generalized function on every space $S_{\alpha}^{\beta}$ such that $\alpha>0, \beta>1$, and the relations

$$
\sum_{k} L^{k} k !\left|d_{2 k}\right| w_{I R}(r)^{k} \leq C_{L, \epsilon} e^{\epsilon r^{1 / \alpha}}, \quad \sum_{k} L^{k} k !\left|d_{2 k}\right| w_{U V}(t)^{k} \leq C_{L, \epsilon} e^{\epsilon t^{-1 /(\beta-1)}}
$$

hold for an arbitrarily large $L>0$ and an arbitrarily small $\epsilon>0$.

This theorem follows immediately from Theorem 3 of [19] because (25) implies the inequality

$$
\inf _{t>0} e^{s t} \sum_{k} L^{k} k !\left|d_{2 k}\right| w_{U V}(t)^{k} \leq C_{L, \epsilon} \exp \left[\beta(\epsilon /(\beta-1))^{(\beta-1) / \beta} s^{1 / \beta}\right] .
$$

It is straightforward to verify that in the case $\alpha>1$, the sum of the series (20) satisfies the usual Wightman axioms (except positivity). For $0<\alpha<1$, we have the following theorem strengthening the results of [12].

Theorem 7. Under the conditions of Theorem [6, the Wightman functions of the field $\varphi(x)=\sum_{k=0}^{\infty} d_{k}: \phi^{k}:(x)$ satisfy the requirements $\mathrm{W} 1-\mathrm{W} 4$ including the generalized spectral condition. 
Proof. The only nontrivial point is to check the fulfilment of the generalized spectral condition. The expression for the $n$-point vacuum expectation value of the field $\varphi$ given by the Wick theorem is a power series in $n(n-1) / 2$ variables $w\left(x_{j}-x_{m}\right)$ and can be written as

$$
\left\langle\Psi_{0}, \varphi\left(x_{1}\right) \ldots \varphi\left(x_{n}\right) \Psi_{0}\right\rangle=\sum_{K} D_{K} w^{K},
$$

where $K$ is an integer-valued vector with nonnegative components $k_{j m}, 1 \leq j<m \leq n$, and $w^{K}(x)$ is the boundary value of the function $\mathbf{w}^{K}(z)=\prod_{j<m} \mathbf{w}\left(z_{j}-z_{m}\right)^{k_{j m}}$ analytic in the tubular domain $\left\{z \in \mathbb{C}^{d n}: z_{j}-z_{m} \in \mathbb{R}^{d}+i \mathbb{V}_{-}, 1 \leq j<m \leq n\right\}$. The usual combinatorial analysis related to the Wick theorem shows that

$$
D_{K}=\frac{\kappa !}{K !} \prod_{1 \leq j \leq n} d_{\kappa_{j}}
$$

where $\kappa_{j}=k_{1 j}+\ldots+k_{j-1, j}+k_{j, j+1}+\ldots+k_{j n}$ is the total number of pairings in the given term of the series that involve the argument $x_{j}$, and we follow the usual convention

$$
K !=\prod_{j<m} k_{j m} !, \quad \kappa !=\prod_{1 \leq j \leq n} \kappa_{j} ! .
$$

Correspondingly, the $n$-point Wightman function expressed in terms of the difference variables is given by

$$
W_{n-1}(\xi)=\sum_{K} D_{K} W^{K}(\xi),
$$

where $W^{K}(\xi)$ is the boundary value of the function $\mathbf{W}^{K}(\zeta)=\prod_{j<m} \mathbf{w}\left(\zeta_{j}+\ldots+\zeta_{m-1}\right)^{k_{j m}}$ analytic in the domain $\mathbb{R}^{d(n-1)}+i \mathbb{V}_{-}^{n-1}$. To prove the theorem, it is sufficient to establish that the series $\sum_{K} D_{K} \mathbf{W}^{K}$ converges unconditionally in $\mathcal{A}_{\alpha}^{\beta}\left(\mathbb{V}_{-}, \ldots, V_{-}\right)$. Indeed, in this case, Theorem $\square$ shows that $\hat{W}_{n-1}$, which is the inverse Laplace transform of the sum of this series, belongs to $s_{\beta}^{\prime \alpha}\left(\mathbb{V}_{+}, \ldots, \mathbb{V}_{+}\right)$, i.e., the generalized spectral condition is satisfied. Since $\mathcal{A}_{\alpha}^{\beta}$ is complete, it suffices to verify that

$$
\sum_{K}\left|D_{K}\right|\left\|\mathbf{W}^{K}\right\|_{\epsilon, V_{1}, \ldots, V_{n-1}}<\infty
$$

for any $\epsilon>0$ and any cones $V_{1}, \ldots, V_{n-1}$ compact in $\mathbb{V}_{-}$. Let $V$ be the closed convex hull of the union $V_{1} \cup \ldots \cup V_{n-1}$. The cone $V$ is the second dual cone of $V_{1} \cup \ldots \cup V_{n-1}$ and, therefore, is a compact subcone of $\mathbb{V}_{-}$(because if $V \Subset U$ and $U$ is an open cone, then $\left.U^{*} \subset \operatorname{int} V^{*}\right)$. Obviously, $\eta_{j}+\ldots+\eta_{m-1} \in V$ for any $\eta=\left(\eta_{1}, \ldots, \eta_{n-1}\right) \in V_{1} \times \ldots \times V_{n-1}$ and for any $1 \leq j<m \leq n$. Further, there is a $\lambda>0$ such that

$$
\left|\eta_{j}+\ldots+\eta_{m-1}\right| \geq \lambda\left(\left|\eta_{j}\right|+\ldots+\left|\eta_{m-1}\right|\right)
$$

for all $\eta \in \overline{\mathbb{V}}_{-}$and $j<m$. Indeed, for fixed $j$ and $m$, (28) is fulfilled if we take

$$
\lambda=\lambda_{j m}=\inf _{\left(\eta_{j}, \ldots, \eta_{m-1}\right) \in \overline{\mathbb{V}}_{-}^{m-j},\left|\eta_{j}\right|+\ldots+\left|\eta_{m-1}\right|=1}\left|\eta_{j}+\ldots+\eta_{m-1}\right| .
$$


By the convexity of $\overline{\mathbb{V}}_{-}$, we have $\eta_{j}+\ldots+\eta_{m-1}=0$ if and only if $\eta_{j}=\ldots=\eta_{m-1}=0$. This implies that $\lambda_{j m}>0$ because the infimum is taken over a compact set. So we can set $\lambda=\min _{j<m} \lambda_{j m}$. By (23), (28), and the monotonicity of $w_{I R}$ and $w_{U V}$, for $\zeta \in \mathbb{R}^{d(n-1)}+i V_{1} \times \ldots \times V_{n-1}$ we have

$$
\left|\mathbf{W}^{K}(\zeta)\right| \leq(n+1)^{|K|} C^{|K|}\left(1+w_{I R}(2 n|\zeta|)^{|K|}+\sum_{i=1}^{n-1} w_{U V}\left(\lambda\left|\eta_{i}\right|\right)^{|K|}\right),
$$

where $|K|=\sum_{j<m} k_{j m}$. The condition (24) and the inequalities $|K| ! / K ! \leq(n(2 n-$ 1) $)^{|K|}$ and $\kappa ! \leq|\kappa| ! \leq 4^{|K|}(|K| !)^{2}$ following from the well-known properties of polynomial coefficients yield

$$
\left|D_{K}\right| \leq A^{\prime} h^{\prime|K|}|K| !\left|d_{2|K|}\right|,
$$

where the constant $h^{\prime}$ depends on $n$. If $w_{I R}$ and $w_{U V}$ are not both identically zero (which is assumed), then (25) implies that for any $L>0$, there is a $\tilde{C}_{L}$ such that

$$
k !\left|d_{2 k}\right| \leq \tilde{C}_{L} L^{-k}, \quad k=0,1 \ldots
$$

Using (25), (29), (30), (31), and Definition 3, we obtain

$$
\left|D_{K}\right|\left\|\mathbf{W}^{K}\right\|_{\epsilon, V_{1}, \ldots, V_{n-1}} \leq C_{L, \epsilon}^{\prime}\left((n+1) C h^{\prime} / L\right)^{|K|} .
$$

This proves (27) because the number of multi-indices $K$ with fixed $|K|$ depends polynomially on $|K|$ and $L$ is arbitrarily large. The theorem is proved.

\section{Conclusion}

We see that the proposed formulation of the spectral condition offers a means for a reasonable generalization of a considerable part of the Wightman-type formalism to quantum fields with highly singular infrared behavior. In particular, gauge-dependent quark fields, which were claimed in 20] to be ill-defined mathematical objects, can be treated in this enlarged axiomatic framework. This situation is somewhat analagous to that in nonlocal QFT, where the corresponding generalization of local commutativity ensures the preservation of the PCT-symmetry [21] and the spin-statistics relation [22, i.e., those basic physical results that are commonly believed to be consequences of locality.

In this paper, we have made no attempt to derive an appropriate extension to infrared singular fields of the Osterwalder-Schrader linear growth estimates which also ensure the reconstruction of Wightman functions from Schwinger functions and which proved to be effective in constructive QFT. At first glance, there are no obstacles for obtaining such a generalization provided the positivity condition is kept. However, this condition is violated for all relevant examples of infrared singular quantum fields. For this reason, we confined our consideration to the indefinite metric case. 
We conclude by a remark on how a notion analogous to that of a strong carrier cone of an analytic functional can be introduced in the framework of Fourier hyperfunctions (i.e., functionals defined on $S_{1}^{1}$ ) which is universal for local QFT [23, 24]. The construction given below is parallel to that of Section 2

Definition $\mathbf{1}^{\prime}$. Let $U$ be an open set in $\mathbb{R}^{k}$. The Banach space $S_{1, B}^{1, A}(U)$ consists of functions analytic in the $1 / A$-neighborhood $U_{1 / A}$ of $U$ in $\mathbb{C}^{k}$ and having the finite norm

$$
\|f\|_{U, A, B}=\sup _{w \in U_{1 / A}}|f(w)| \exp (|p / B|)
$$

The space $S_{1}^{1}(U)$ is defined to be the inductive limit $\lim _{A, B>0} S_{1, B}^{1, A}(U)$.

Let $\mathcal{R}^{k}$ be the radial compactification of $\mathbb{R}^{k}$. For $\mathcal{U} \subset \mathcal{R}^{k}$, we denote by $\tilde{\mathcal{U}}$ the set $\mathcal{U} \cap \mathbb{R}^{k}$. A compact set $\mathcal{K} \subset \mathcal{R}^{k}$ is said to be a carrier of a functional $u \in S_{1}^{\prime 1}\left(\mathbb{R}^{k}\right)$ if $u$ has a continuous extension to the space $s_{1}^{1}(\mathcal{K})=\lim _{\longrightarrow} S_{1}^{1}(\tilde{\mathcal{U}})$, where $\mathcal{U}$ runs over all open neighborhoods of $\mathcal{K}$ in $\mathcal{R}^{k}$. The following definition is an analogue of Definition 2.

Definition $2^{\prime}$. Let $\mathcal{K}_{1}, \ldots, \mathcal{K}_{n}$ be compact sets in $\mathcal{R}^{k_{1}}, \ldots, \mathcal{R}^{k_{n}}$ respectively. The functional $u \in S_{1}^{\prime 1}\left(\mathbb{R}^{k_{1}+\ldots+k_{n}}\right)$ is said to be carried by the family of sets $\mathcal{K}_{1}, \ldots, \mathcal{K}_{n}$ if $u$ has a continuous extension to the space $s_{1}^{1}\left(\mathcal{K}_{1}, \ldots, \mathcal{K}_{n}\right)=\lim _{\mathcal{U}_{1}, \ldots \mathcal{U}_{n}} S_{1}^{1}\left(\tilde{\mathcal{U}}_{1} \times \ldots \times \tilde{\mathcal{U}}_{n}\right)$, where the inductive limit is taken over all open neighborhoods $\mathcal{U}_{1}, \ldots, \mathcal{U}_{n}$ of the sets $\mathcal{K}_{1}, \ldots, \mathcal{K}_{n}$ respectively.

For $K \subset \mathbb{R}^{k}$, we denote by $\hat{K}$ the closure of $K$ in $\mathcal{R}^{k}$. Let $K_{1}, \ldots K_{n}$ be closed sets in $\mathbb{R}^{k_{1}}, \ldots, \mathbb{R}^{k_{n}}$ respectively, and let $K=K_{1} \times \ldots \times K_{n}$. The following example shows that the space $s_{1}^{\prime 1}(\hat{K})$ is, in general, different from $s_{1}^{11}\left(\hat{K}_{1}, \ldots, \hat{K}_{n}\right)$.

Example $1^{\prime}$. Let $k_{1}=k_{2}=1, K_{1}=\overline{\mathbb{R}}_{+}, K_{2}=\{0\}$, and $K=\overline{\mathbb{R}}_{+} \times\{0\}$. Clearly, $s_{1}^{\prime 1}\left(\hat{K}_{1}, \hat{K}_{2}\right) \subset s_{1}^{\prime 1}(\hat{K})$. In this case, we can assume that $\tilde{\mathcal{U}}_{1,2}$ in Definition $2^{\prime}$ are just the $\varepsilon$ neighborhoods of $\overline{\mathbb{R}}_{+}$and $\{0\}$ respectively. If $\varepsilon<1 / 2$, then the sequence $g_{n}(p)=p_{2}^{n} e^{-p_{1}}$ converges to zero in every space $S_{1, B}^{1, A}\left(\tilde{\mathcal{U}}_{1} \times \tilde{\mathcal{U}}_{2}\right)$ with $A>2$ and $B>1$ and therefore is bounded in $s_{1}^{1}\left(\hat{K}_{1}, \hat{K}_{2}\right)$. If the sequence $g_{n}$ were bounded in the DFS-space $s_{1}^{1}(\hat{K})$, then it would be bounded in some space $S_{1, B}^{1, A}(\tilde{\mathcal{U}})$, where $\mathcal{U}$ is an open neighborhood of $\hat{K}$. However, any such $\mathcal{U}$ contains the ray $r_{\lambda}=\left\{\left(p_{1}, p_{2}\right): p_{1} \geq 0, p_{2}=\lambda p_{1}\right\}$ with some $\lambda>0$ and, therefore, we have $\left\|g_{n}\right\|_{\tilde{\mathcal{U}}, A, B} \geq \sup _{p \in r_{\lambda}}\left|g_{n}(p)\right|=\lambda^{n} n^{n} e^{-n}$. Thus, the sequence $g_{n}$ is unbounded in $s_{1}^{1}(\hat{K})$ and there is an $u \in s_{1}^{\prime 1}(\hat{K})$ such that the number sequence $\left|u\left(g_{n}\right)\right|$ is unbounded (because any weakly bounded set in a locally convex space is bounded). Obviously, $u$ does not belong to $s_{1}^{\prime 1}\left(\hat{K}_{1}, \hat{K}_{2}\right)$ and so the latter is different from $s_{1}^{\prime 1}(\hat{K})$.

This distinction may be essential for hyperfunction QFT, where the spectral condition can be formulated in two alternative ways. One can require either that $\hat{W}_{n} \in$ $s_{1}^{\prime 1}\left(\hat{\mathbb{V}}_{+}^{n}\right)$ as in 23, 24] or that $\hat{W}_{n} \in s_{1}^{\prime 1}\left(\hat{\mathbb{V}}_{+}, \ldots, \hat{\mathbb{V}}_{+}\right)$. It would be worthwhile to examine the second condition from the viewpoint of the Euclidean formulation of hyperfunction QFT, but this is beyond the scope of the present paper. 


\section{Acknowledgments}

The author is grateful to M. A. Soloviev for helpful and stimulating discussions. This research was supported by the Russian Foundation for Basic Research (Grants No. 02-01-00556 and No. 00-15-96566) and INTAS (Grant No. 99-1-590).

\section{Appendix A. Proof of Lemma 1 for $\alpha=0$.}

Lemma 11. Let $Q_{1}, Q_{2}$ be nonempty cones in $\mathbb{R}^{k}$ and let $h_{1}, h_{2}$ be $C^{\infty}$-functions on $\mathbb{C}^{k}$ such that $f=h_{1}+h_{2}$ is analytic in $\mathbb{C}^{k}$. If the norms $\left\|h_{1}\right\|_{Q_{1}, A, B},\left\|h_{2}\right\|_{Q_{2}, A, B}$, and $\left\|\partial h_{1} / \partial \bar{w}_{j}\right\|_{Q_{1} \cup Q_{2}, A, B}$ (given by (4) with $\alpha=0$ ) are finite for some $A, B>0$, then one can find $f_{1,2} \in S_{\beta}^{0}\left(Q_{1,2}\right)$ such that $f=f_{1}+f_{2}$.

Let us derive Lemma 1 from Lemma 11, Let $\chi \in C_{0}^{\infty}\left(\mathbb{R}^{k_{1}}\right)$ satisfy $\int_{\mathbb{R}^{k_{1}}} \chi\left(p^{\prime}\right) \mathrm{d} p^{\prime}=1$. We set $g_{0}\left(w^{\prime}\right)=\chi\left(\operatorname{Re} w^{\prime}\right)$ and define $g_{1,2}$ by ([6) . The functions $g_{1,2}(w)$ as well as their derivatives $\partial g_{1,2} / \partial \bar{w}_{j}, j=1, \ldots, k_{1}+k_{2}$, satisfy the estimate (7) with $\alpha=0$ for any $A_{0}, B_{0}>0$. Therefore, setting $Q_{1,2}=\left(U \cup U_{1,2}\right) \times V$ and repeating the proof for nonzero $\alpha$, we conclude that the norms $\left\|f g_{2}\right\|_{Q_{1}, A, B},\left\|f g_{1}\right\|_{Q_{2}, A, B},\left\|f \partial g_{2} / \partial \bar{w}_{j}\right\|_{Q_{1}, A, B}$, and $\left\|f \partial g_{1} / \partial \bar{w}_{j}\right\|_{Q_{2}, A, B}$ are finite for $A, B$ sufficiently large. Moreover, since $g_{1}+g_{2}=1$ and $\partial g_{1} / \partial \bar{w}_{j}=-\partial g_{2} / \partial \bar{w}_{j}$, we have $\left\|f \partial g_{2} / \partial \bar{w}_{j}\right\|_{Q_{1} \cup Q_{2}, A, B}<\infty$. Thus, $h_{1,2}=f g_{2,1}$ satisfy the conditions of Lemma 11] because $\partial h_{1} / \partial \bar{w}_{j}=f \partial g_{2} / \partial \bar{w}_{j}$ in view of the analyticity of $f$. Lemma 1 is proved.

Proof of Lemma 11. Let $h$ be a measurable function on $\mathbb{C}^{k}$ and $U$ be a nonempty cone in $\mathbb{R}^{k}$. For $a, b>0$ sufficiently large, from Definition (41) it follows that

$$
C\|h\|_{U, A, B} \geq\|h\|_{U, a, b}^{\prime}=\left[\int_{\mathbb{C}^{k}}|h(w)|^{2} \exp \left(-\rho_{U, a, b}(w)\right) \mathrm{d} \lambda(w)\right]^{1 / 2},
$$

where $\rho_{U, a, b}(p+i q)=-\sum_{j=1}^{k}\left|p_{j} / b\right|^{1 / \beta}+a \inf _{p^{\prime} \in U} \sum_{j=1}^{k}\left|p_{j}-p_{j}^{\prime}\right|+a \sum_{j=1}^{k}\left|q_{j}\right|, \mathrm{d} \lambda$ is the Lebesgue measure on $\mathbb{C}^{k}$, and $C$ is a constant independent of $h$. If $h$ is analytic, then using Cauchy's integral formula, one can prove [10] the converse statement, i.e., if $\|h\|_{U, a, b}^{\prime}<\infty$ for some $a, b>0$, then $h \in S_{\beta}^{0}(U)$.

Suppose there is a locally integrable function $\psi$ on $\mathbb{C}^{k}$ which has the finite norm $\|\psi\|_{Q_{1} \cup Q_{2}, a_{1}, b_{1}}^{\prime}$ for some $a_{1}, b_{1}>0$ and satisfies, as a generalized function, the inhomogeneous Cauchy-Riemann equations

$$
\frac{\partial \psi}{\partial \bar{w}_{j}}=\eta_{j}, \quad j=1, \ldots, k
$$

where $\eta_{j}=\partial h_{1} / \partial \bar{w}_{j}$. Then $f_{1}=h_{1}-\psi$ and $f_{2}=h_{2}+\psi$ satisfy, as generalized functions, the homogeneous equations $\partial f_{1,2} / \partial \bar{w}_{j}=0$ and, consequently, are ordinary analytic functions. Moreover, in view of (32) we have $\left\|f_{1,2}\right\|_{Q_{1,2}, a, b}^{\prime}<\infty$ for $a \geq a_{1}$, $b>b_{1}$ sufficiently large, i.e., $f_{1,2} \in S_{\beta}^{0}\left(Q_{1,2}\right)$. The following lemma allows to apply 
the Hörmander's $L_{2}$-estimates [25] to prove of the existence of a function $\psi$ with the specified properties.

Lemma 12. For any $a, b>0$ and any nonempty cone $U \subset \mathbb{R}^{k}$ there are a plurisubharmonic function $\rho$ on $\mathbb{C}^{k}$, positive numbers $a^{\prime}, b^{\prime}$, and a constant $H$ such that $\rho_{U, a, b}-H \leq \rho \leq \rho_{U, a^{\prime}, b^{\prime}}$.

Supposing Lemma 12 is proved, we finish the derivation of Lemma 11. Let $U=Q_{1} \cup$ $Q_{2}$. By (32) and the condition of Lemma 11, there are $a, b>0$ such that $\left\|\eta_{j}\right\|_{U, a, b}^{\prime}<\infty$. According to Theorem 4.4.2 of [25] there exists a solution $\psi$ of equations (33) such that

$$
2 \int_{\mathbb{C}^{k}}|\psi|^{2} e^{-\rho}\left(1+\|w\|^{2}\right)^{-2} \mathrm{~d} \lambda(w) \leq \sum_{j=1}^{k} \int_{\mathbb{C}^{k}}\left|\eta_{j}\right|^{2} e^{-\rho} \mathrm{d} \lambda(w),
$$

where $\|w\|=\left(\left|w_{1}\right|^{2}+\ldots+\left|w_{k}\right|^{2}\right)^{1 / 2}$. By Lemma 12, the integrals in the right-hand side are bounded by $e^{H}\left(\left\|\eta_{j}\right\|_{U, a, b}^{\prime}\right)^{2}$ and, therefore, are convergent. Estimating $e^{-\rho}$ in the lefthand side of (34) from below by the function $e^{-\rho_{U, a^{\prime}, b^{\prime}}}$, we conclude that $\|\tilde{\psi}\|_{U, a^{\prime}, b^{\prime}}^{\prime}<\infty$, where $\tilde{\psi}=\left(1+\|w\|^{2}\right)^{-1} \psi$. To complete the proof, it remains to note that $\|\psi\|_{U, a_{1}, b_{1}}^{\prime} \leq$ $C\|\tilde{\psi}\|_{U, a^{\prime}, b^{\prime}}^{\prime}$ for $a_{1}>a^{\prime}, b_{1}>b^{\prime}$.

Proof of Lemma [12 is essentially contained in the derivation of Theorem 5 of [11]. We assume $0<a<1 / 2 e k$; to pass to the general case, it suffices to make a rescaling of the arguments. Let $\sigma=e k a$. By Lemma 4 of [1], there are a sequence $\varphi_{N}(w) \in S_{\beta}^{0}(\mathbb{R})$ and constants $A, B>0$ independent of $N$ such that

$$
\begin{aligned}
& \left|\varphi_{N}(w)\right| \leq A \exp \left(|q|-|p / b|^{1 / \beta}\right), \quad w=p+i q \in \mathbb{C}, \\
& \ln \left|\varphi_{N}(i q)\right| \geq \sigma|q|, \\
& \ln \left|\varphi_{N}(w)\right| \leq|q|-N \ln ^{+}(\sigma|p| / N)+B
\end{aligned}
$$

where $\ln ^{+} r=\max (0, \ln r)$. Let $a^{\prime} \geq 2$ and let

$$
\tilde{\rho}(w)=\sup _{\kappa \in \mathbb{R}^{k}, N}\left\{\Phi_{N}(w-\kappa)+M_{N}(\kappa)\right\}, \quad M_{N}(\kappa)=\inf _{w \in \mathbb{C}^{k}}\left\{-\Phi_{N}(w-\kappa)+\rho_{U, a^{\prime}, b}(w)\right\},
$$

where $\Phi_{N}(w)=2 \sum_{j=1}^{k} \ln \left|\varphi_{N}\left(w_{j}\right)\right|$. Obviously, we have $\tilde{\rho} \leq \rho_{U, a^{\prime}, b}$. Since functions $\Phi_{N}$ are plurisubharmonic, $\rho(w)=\varlimsup_{w^{\prime} \rightarrow w} \tilde{\rho}(w)$ is also a plurisubharmonic function, see [26], Sec. II.10.3. In view of the continuity of $\rho_{U, a^{\prime}, b}$ we have $\tilde{\rho} \leq \rho \leq \rho_{U, a^{\prime}, b}$ and it remains to show that $\tilde{\rho} \geq \rho_{U, a, b}-H$. From (35) and the inequality $\left|p_{j}-\kappa_{j}\right|^{1 / \beta} \geq\left|p_{j}\right|^{1 / \beta}-\left|\kappa_{j}\right|^{1 / \beta}$, it follows that

$$
-\Phi_{N}(w-\kappa) / 2-\sum_{j}\left|p_{j} / b\right|^{1 / \beta}+\sum_{j}\left|q_{j}\right| \geq-k \ln A-\sum_{j}\left|\kappa_{j} / b\right|^{1 / \beta}
$$

and hence $M_{N}(\kappa) \geq-k \ln A-\sum_{j}\left|\kappa_{j} / b\right|^{1 / \beta}+L_{N}(\kappa)$, where

$$
L_{N}(\kappa)=\inf _{w \in \mathbb{C}^{k}}\left\{-\Phi_{N}(w-\kappa) / 2+\inf _{p^{\prime} \in U} \sum_{j}\left|p_{j}-p_{j}^{\prime}\right|+\sum_{j}\left|q_{j}\right|\right\} .
$$


Therefore, estimating the supremum in (38) from below by the value of the function at $\kappa=p=\operatorname{Re} w$ and taking (36) and the inequality $2 \sigma>a$ into account, we find that

$$
\tilde{\rho}(w) \geq a \sum_{j}\left|q_{j}\right|-\sum_{j}\left|p_{j} / b\right|^{1 / \beta}+\sup _{N} L_{N}(p)-k \ln A .
$$

Thus, it suffices to show that $\sup _{N} L_{N}(p) \geq a \inf _{p^{\prime} \in U} \sum_{j}\left|p_{j}-p_{j}^{\prime}\right|-C$. Passing to the Euclidean norm $\|p\|$, using the elementary inequalities $\sum_{j} \ln ^{+}\left|p_{j}\right| \geq \ln ^{+}(\|p\| / \sqrt{k})$ and $\|p\| \leq \sum_{j}\left|p_{j}\right|$, and estimating $\Phi_{N}$ by (37), we conclude that

$$
L_{N}(p) \geq-k B+\inf _{\lambda \in \mathbb{R}^{k}}\left\{N \ln ^{+}(\sigma\|\lambda\| / N \sqrt{k})+\delta_{U}(p+\lambda)\right\}
$$

where $\delta_{U}(p)=\inf _{p^{\prime} \in U}\left\|p-p^{\prime}\right\|$. Estimating $\delta_{U}(p+\lambda)$ from below by $\max \left(\delta_{U}(p)-\|\lambda\|, 0\right)$ and calculating the infimum with respect to $\lambda$ yield $L_{N}(p) \geq N \ln ^{+}\left(\sigma \delta_{U}(p) / N \sqrt{k}\right)-k B$. Let $\delta_{U}(p) \geq e \sqrt{k} / \sigma$ and let $N_{0}$ be the integer part of $\sigma \delta_{U}(p) / e \sqrt{k}$. In view of the inequality $\sum_{j}\left|p_{j}\right| \leq \sqrt{k}\|p\|$ we find that

$$
\sup _{N} L_{N}(p) \geq L_{N_{0}} \geq \frac{\sigma \delta_{U}(p)}{e \sqrt{k}}-1-k B \geq a \inf _{p^{\prime} \in U} \sum_{j}\left|p_{j}-p_{j}^{\prime}\right|-C .
$$

Since $L_{N}(p) \geq-k \ln A$ by (35), this inequality holds for all $p \in \mathbb{R}^{k}$ with a new constant $C$. The lemma is proved.

\section{Appendix B. Proof of Lemma 4}

By Lemma 2 of [14, the spaces $L^{(1,2)}$ are representable as inductive limits of sequences of Banach spaces $L_{k}^{(1,2)}$ with injective connecting mappings $u_{k m}^{(1,2)}: L_{k}^{(1,2)} \rightarrow L_{m}^{(1,2)}$, $1 \leq k \leq m$, which take unit balls in $L_{k}^{(1,2)}$ to compact subsets of $L_{m}^{(1,2)}$. Let $M_{k}=$ $L_{k}^{(1)} \otimes_{i} L_{k}^{(2)}=L_{k}^{(1)} \otimes_{\pi} L_{k}^{(2)}$ and $M=\lim _{k} M_{k}$. We denote by $u_{k}^{(1,2)}$ and $u_{k}$ the canonical embeddings of $L_{k}^{(1,2)}$ into $L^{(1,2)}$ and of $M_{k}$ into $M$ respectively. One can identify the space $M$ with $L^{(1)} \otimes_{i} L^{(2)}$ using the canonical separately continuous bilinear mapping from $L^{(1)} \times L^{(2)}$ into $M$ which is uniquely determined by the relations

$$
u_{k}^{(1)}\left(x_{1}\right) \otimes u_{k}^{(2)}\left(x_{2}\right)=u_{k}\left(x_{1} \otimes x_{2}\right), \quad x_{1,2} \in L_{k}^{(1,2)} .
$$

To prove the lemma, it suffices to show that this mapping is continuous. Let $V$ be an absolutely convex neighborhood of the origin in $M$. Set $V_{k}=u_{k}^{-1}(V)$. We shall construct sequences of absolutely convex neighborhoods $V_{k}^{(1,2)}$ of the origin in $L_{k}^{(1,2)}$ such that

(i) $u_{k m}^{(1,2)}\left(V_{k}^{(1,2)}\right) \subset V_{m}^{(1,2)}$ for $m>k$;

(ii) $V_{k}^{(1)} \otimes V_{k}^{(2)} \subset V_{k}, k=1,2 \ldots$; 
(iii) The set $u_{k m}^{(1,2)}\left(V_{k}^{(1,2)}\right)$ is compact in $L_{m}^{(1,2)}$ for $m>k$.

The sets $V^{(1,2)}=\bigcup_{k=1}^{\infty} u_{k}^{(1,2)}\left(V_{k}^{(1,2)}\right)$ are neighborhoods of the origin in $L^{(1,2)}$ because they are absolutely convex (in view of (i)) and $\left[u_{k}^{(1,2)}\right]^{-1}\left(V^{(1,2)}\right)$ contain $V_{k}^{(1,2)}$. Moreover, by (39) and property (ii), we have $V^{(1)} \otimes V^{(2)} \subset V$, i.e., the mapping $(x, y) \rightarrow x \otimes y$ from $L^{(1)} \times L^{(2)}$ to $M$ is continuous.

We construct the sequences $V_{k}^{(1,2)}$ by induction. Let $\tilde{V}_{k}$ denote the inverse image of $V_{k}$ under the canonical bilinear mapping from $L_{k}^{(1)} \times L_{k}^{(2)}$ to $M_{k}$. Let $V_{1}^{(1,2)}$ be closed balls in $L_{1}^{(1,2)}$ such that $V_{1}^{(1)} \times V_{1}^{(2)} \subset \tilde{V}_{1}$. Suppose $V_{1}^{(1,2)}, \ldots, V_{k}^{(1,2)}$ satisfying (i)-(iii) are constructed. The compactum $u_{k, k+1}^{(1)}\left(V_{k}^{(1)}\right) \times u_{k, k+1}^{(2)}\left(V_{k}^{(2)}\right)$ is contained in the open neighborhood of the origin $\tilde{V}_{k+1}$. Hence, there are closed balls $B^{(1,2)}$ in $L_{k+1}^{(1,2)}$ such that $\left[u_{k, k+1}^{(1)}\left(V_{k}^{(1)}\right)+B^{(1)}\right] \times\left[u_{k, k+1}^{(2)}\left(V_{k}^{(2)}\right)+B^{(2)}\right] \subset \tilde{V}_{k+1}$. Set $V_{k+1}^{(1,2)}=u_{k, k+1}^{(1,2)}\left(V_{k}^{(1,2)}\right)+B^{(1,2)}$. Conditions (i) and (ii) are obviously satisfied. If $m>k+1$, then $u_{k+1, m}^{(1,2)}\left(V_{k+1}^{(1,2)}\right)$ is the sum of the compact sets $u_{k, m}^{(1,2)}\left(V_{k}^{(1,2)}\right)$ and $u_{k+1, m}^{(1,2)}\left(B^{(1,2)}\right)$ and, therefore, is also compact.

To prove the second statement of the lemma it suffices to note that $i$ - and $\pi$ topologies coincide on the tensor product of the Fréchet spaces $L^{(1) \prime}$ and $L^{(2) \prime}$ and that $\left(L^{(1)} \hat{\otimes}_{\pi} L^{(2)}\right)^{\prime}=L^{(1) \prime} \hat{\otimes}_{\pi} L^{(2) \prime}$ for arbitrary DF-spaces $L^{(1,2)}$ one of which is nuclear (see 15, Chapter IV, Problem 32). The lemma is proved.

\section{References}

[1] J. Glimm, A. Jaffe, Quantum Physics: A functional integral point of view, 2nd ed. Berlin, Heidelberg, New York: Springer-Verlag, 1981.

[2] K. Osterwalder, R. Schrader, Commun. Math. Phys. 31 (1973) 83.

[3] K. Osterwalder, R. Schrader, Commun. Math. Phys. 42 (1975) 281.

[4] G. Morchio, F. Strocchi, Ann. Inst. Henri Poincaré 33A (1980) 251.

[5] N. N. Bogoliubov, A. A. Logunov, A. I. Oksak, and I. T. Todorov, General Principles of Quantum Field Theory, Dordrecht: Kluwer, 1990.

[6] L. Jakobczyk, F. Strocchi, Commun. Math. Phys. 119 (1988) 529.

[7] A. Z. Capri, R. Ferrari, J. Math. Phys. 25 (1984) 141.

[8] U. Moschella, F. Strocchi, Lett. Math. Phys. 24 (1992) 103.

[9] M. A. Soloviev, Theor. Math. Phys. 105 (1995) 1520.

[10] M. A. Soloviev, Lett. Math. Phys. 33 (1995) 49.

[11] M. A. Soloviev, Commun. Math. Phys. 184 (1997) 579. 
[12] A. G. Smirnov, M. A. Soloviev, Theor. Math. Phys. 125 (2000) 1349.

[13] I. M. Gelfand, G. E. Shilov, Generalized functions, Vol. 2. New York: Academic Press, 1964.

[14] H. Komatsu, J. Math. Soc. Japan 19 (1967) 366.

[15] H. Schaefer, Topological Vector Spaces. Berlin, Heidelberg, New York: SpringerVerlag, 1981.

[16] F. Treves, Topological vector spaces, distributions, and kernels. New York-London: Academic Press, 1967.

[17] R. Jost, The General Theory of Quantized Fields. Providence, Rhode Island: Am. Math. Soc. Publ., 1965.

[18] A. G. Smirnov, M. A. Soloviev, "Wick Power Series in Indefinite Metric Field Theories", in "Quantization, Gauge Theory, and Strings" (Proc. of the International Conference dedicated to the memory of Prof. Efim Fradkin, Moscow, Russia, June 5-10, 2000), Vol.2, P. 107-116, Scientific World, Moscow, 2001.

[19] A. G. Smirnov, M. A. Soloviev, Theor. Math. Phys. 123 (2000) 709.

[20] K. D. Rothe, B. Schroer, Nucl. Phys. B172 (1980) 383.

[21] M. A. Soloviev, J. Math. Phys. 39 (1998) 2635.

[22] M. A. Soloviev, Theor. Math. Phys. 121 (1999) 1377.

[23] S. Nagamachi, N. Mugibayashi, Commun. Math. Phys. 46 (1976) 119; Commun. Math. Phys. 49 (1976) 257.

[24] E. Brüning, S. Nagamachi, J. Math. Phys. 30 (1989) 2340.

[25] L. Hörmander, An Introduction to Complex Analysis in Several Variables. Princeton, N.J.: D. van Nostrand Publ. Co., 1966.

[26] V. S. Vladimirov, Methods of the Theory of Functions of Several Complex Variables. Cambridge: MIT Press, 1966. 